\title{
THE CHARACTERIZATION AND ANALYSIS OF IN-VITRO AND ELEVATED TEMPERATURE REPASSIVATION OF Ti-6Al-4V VIA AFM TECHNIQUES
}

\author{
A Thesis \\ presented to \\ the faculty of California Polytechnic State University, \\ San Luis Obispo
}

\begin{abstract}
In Partial Fulfillment
of the Requirements for the Degree

Master of Science in Engineering, with Specialization in Materials Engineering
\end{abstract}

By

Aaron J. Guerrero

May 2010 
(C) 2010

AARON J. GUERRERO

ALL RIGHTS RESERVED 


\section{COMMITTEE MEMBERSHIP}

TITLE:

The characterization and analysis of in-vitro and elevated temperature repassivation of Ti-6Al-4V via AFM techniques

AUTHOR:

DATE SUBMITTED:

COMMITTEE CHAIR:

COMMITTEE MEMBER:

COMMITTEE MEMBER:
Aaron J. Guerrero

May 2010

Trevor Harding, Ph.D

Dept. Chair/ Faculty, Materials Engineering

California Polytechnic State University, San Luis Obispo

William L. Hughes, Ph.D

Faculty, Materials Science \&Engineering

Boise State University, Boise, Idaho

Kevin Baker, MS

Supervisor of Orthopaedic Research Laboratory

Beaumont Hospital, Royal Oaks, Michigan 


\begin{abstract}
The Characterization and Analysis of In-vitro and Elevated Temperature Repassivation of Ti-6Al-4V via AFM Techniques
\end{abstract}

Aaron J Guerrero

Research in the corrosion of orthopaedic implants is a growing research field where implants have been known to show adverse effects in patients who have encountered the unfortunate dissolution of their implants due to corrosion. Once corrosion begins within the body, many adverse biological reactions can occur such as late on-set infections resulting in severe health complications. The focus of this research is specifically related to the problem of late onset infections caused by localized corrosion of orthopaedic implants. In medical implants today the most common form of corrosion protection is the implant materials' ability to impede corrosion through the formation of an oxide layer. This ability to passivate and quickly repassivate a uniform and stable oxide layer dictates how well an orthopaedic implant will survive in-vivo.

To better understand the repassivation of orthopaedic implant materials, research was conducted at the nanoscale via atomic force microscopy (AFM) on anodized Ti-6Al-4V. Using an Asylum Research MFP-3D ${ }^{\mathrm{TM}}$ AFM and AFM lithography techniques, nano scratch test methods were created simulating in-vitro surface repassivation conditions. These nano-scratches were created and characterized in Hank's balanced saline solution (HBSS) with the AFM in contact mode at 1 and $3 \mathrm{~Hz}$ scan rates. HBSS was used as it best simulates the $\mathrm{pH}$, ionic compounds, and constituents that are commonly found in blood. It was discovered that the AFM was successful in creating in-vitro repassivation conditions. However, the ability of the AFM to successfully observe repassivation was limited by the speed of the AFM scanner.

Using the same AFM scratch methods, experiments were performed in air and in-vitro and characterized with AFM conductance measurements at $20,37, \& 45^{\circ} \mathrm{C}$. The conductance measurements were taken using an AFM conductance module and allowed for observations of 
decreasing current measurements over time. The current data was then used to calculate current density, resistivity, conductance, and electron mobility and compared to similar experiments

This study highlights the ability of the AFM to create and characterize repassivation and shows promise in developing further capability to use the AFM for characterization of repassivation on the nanoscale.

Keywords: Orthopaedics, late on-set infections, repassivation, AFM, lithography, conductive measurements. 


\section{ACKNOWLEDGEMENTS}

To my family, thank you for the unconditional love and support throughout my life and education. I am who I am today because of you and I am truly grateful for having such an amazing family. You have encouraged me to work hard and invest in myself by pursuing my educational goals

To my committee members, I would like to express my utmost appreciation for helping me throughout my thesis research. To Kevin Baker, supervisor of Orthopaedic research at Beaumont Hospital in Royal Oak Michigan, for without you, the topic of research would not have been presented. To Dr. William Hughes, thank you for your continued support and commitment as an advisor, mentor, and friend after your departure to Boise State. To Dr. Trevor Harding for being a great advisor, friend, and more importantly the beacon of light that helped navigate this ship into harbor when all was thought to be lost in my research.

I would also like to acknowledge some of my peers from which I received invaluable feedback and support during my thesis research; Matthew Goebel, Matt Lewis, Darin Grandfield, Tedman Tong, Greg Boban, Brian Stahl, Patrick Kuga, Nate Knock, Sean Kaylor, and Steve Meredith. I will always be truly grateful for all your help and support. A special thanks to Dr. Richard Savage for encouraging me to pursue my master's degree, without your words and encouragement, I don't think I would have ever considered it.

Lastly, to all my friends, thank you for all your support and understanding, especially when you realized that by me pursuing my master's meant my social time would become limited. To my roommate Fabio Fortunat, for always being considerate when I needed quiet time to work as well as always willing to listen to me vent about my problems in my research. To Kirsten Thom, for always being supportive and having the ability to bring me back to reality when I was stressed out. Your comforting words, food, and coffee were much appreciated and needed. I thank 
you all for helping me through this. Without all of you, I would have never been able to accomplish this project. 


\section{TABLE OF CONTENTS}

List of Tables .. $\mathbf{X}$

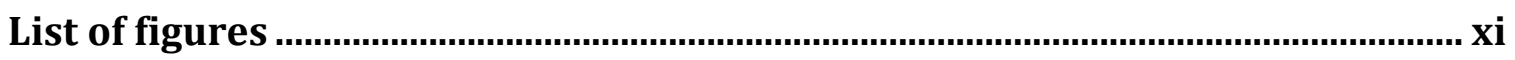

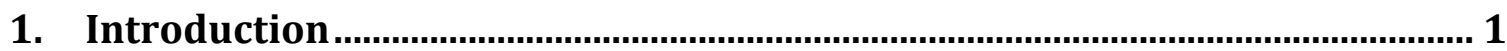

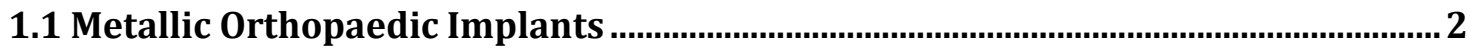

1.2 Orthopaedic Infections

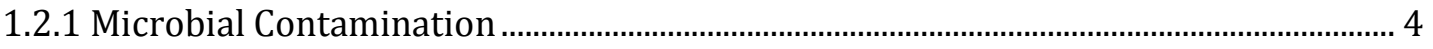

1.2.2 Foreign Body Responses (FBR's)................................................................................ 5

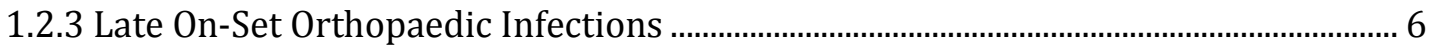

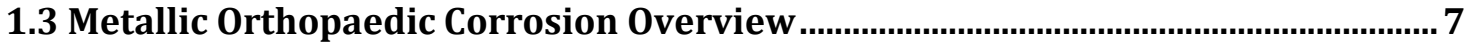

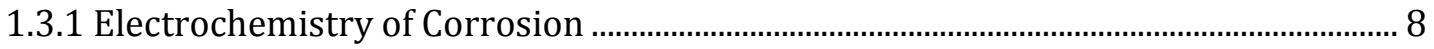

1.3.2 Thermodynamic Driving Force of Corrosion.................................................................... 9

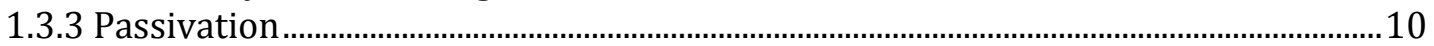

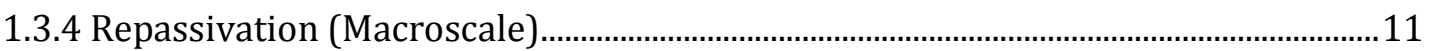

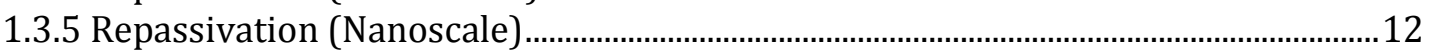

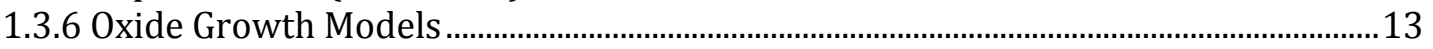

1.3.7 Previous Studies of Orthopaedic Repassivation .............................................................14

1.4 Atomic Force Microscopy (AFM): Method to Investigate Nano-Repassivation .. 16

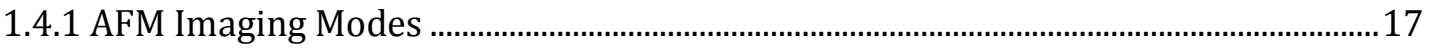

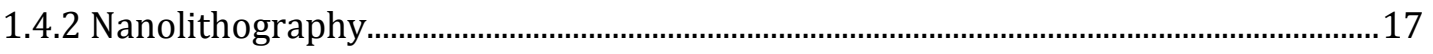

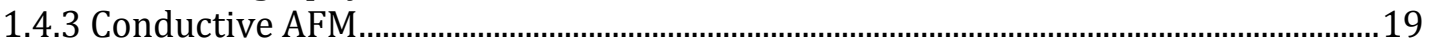

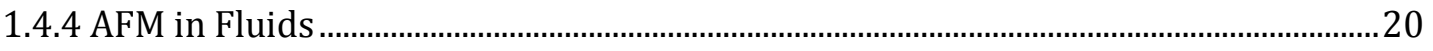

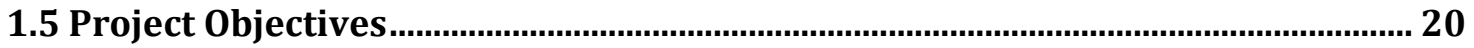

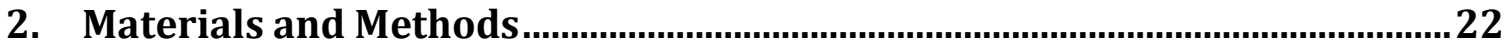

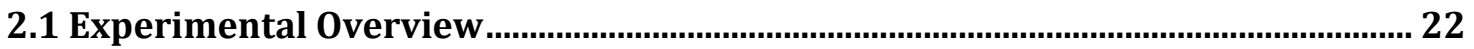

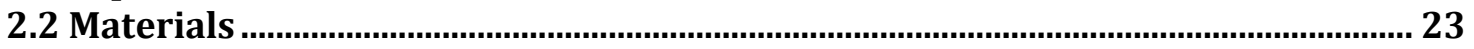

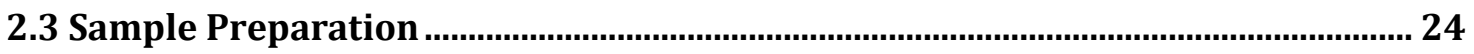

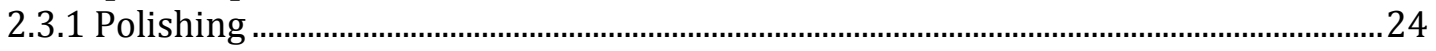

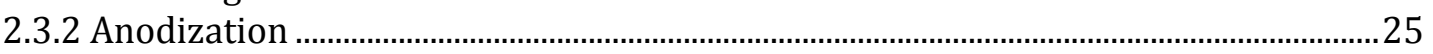

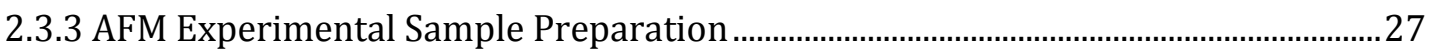

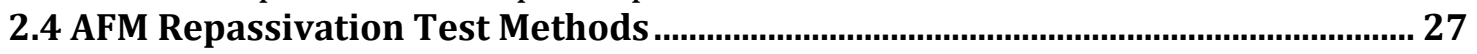

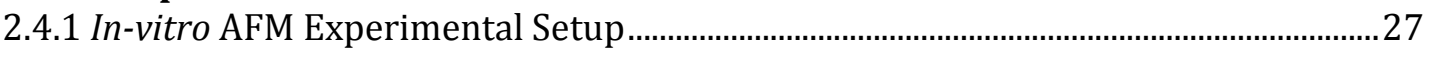

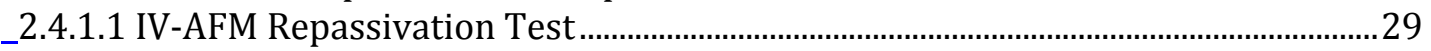

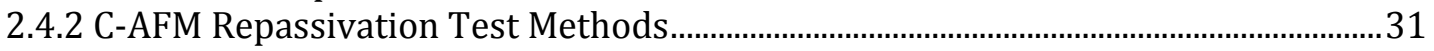

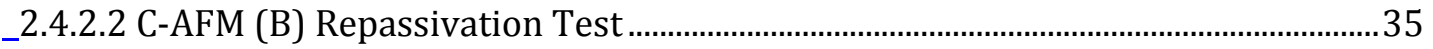

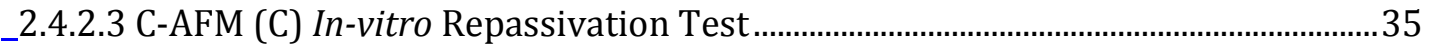

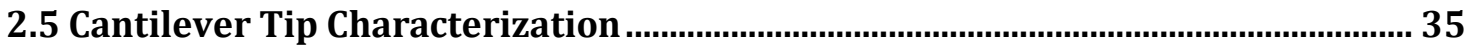

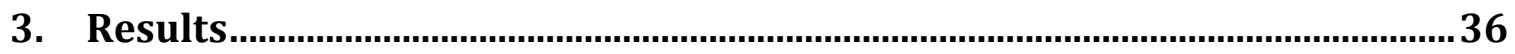

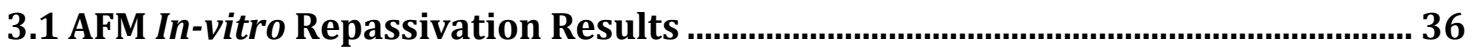

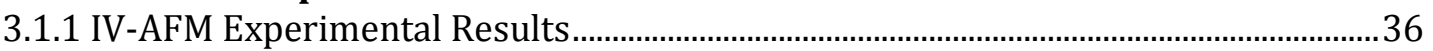

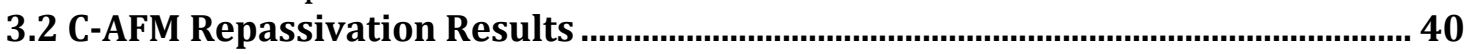

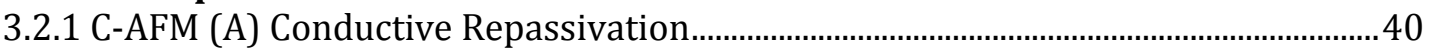

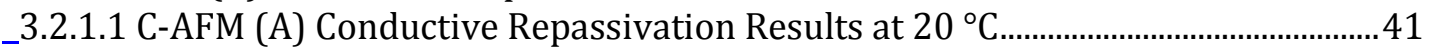

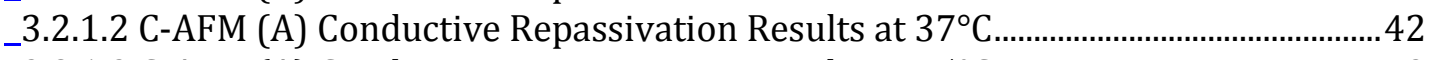

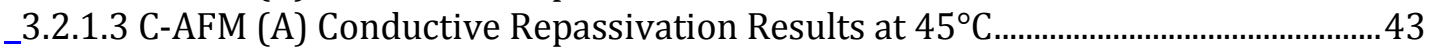

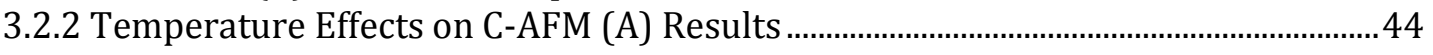

3.2.3 C-AFM (B) Conductive Repassivation Results.............................................................. 45 
3.2.4 C-AFM (C) In-vitro Repassivation Results...................................................................... 47

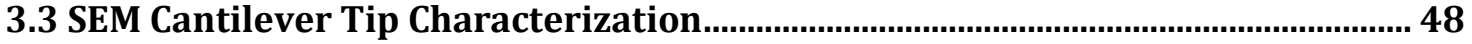

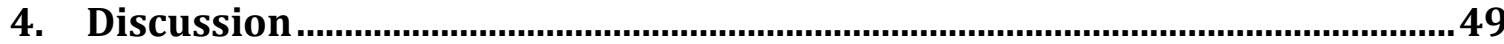

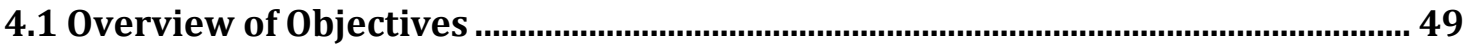

4.2 Evaluation of In-vitro Repassivation via AFM Z-Sensor Data....................................49

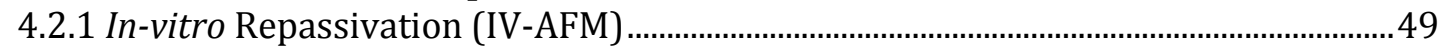

4.3 Evaluation of Repassivation via Conductive AFM Methods................................... 51

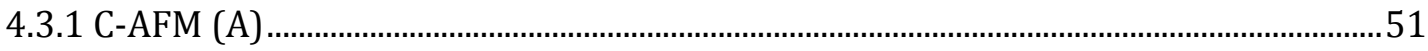

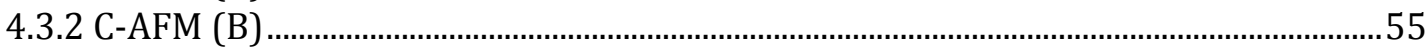

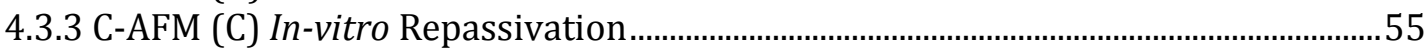

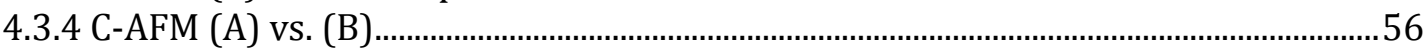

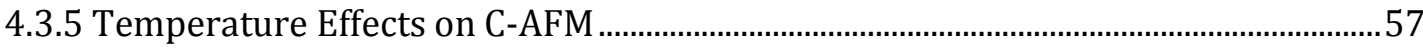

4.4 C-AFM Experiments Compared to Oxide Film Growth Model .................................. 58

5. Conclusion ........................................................................................................60

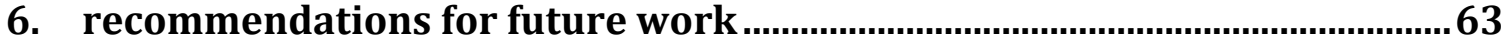

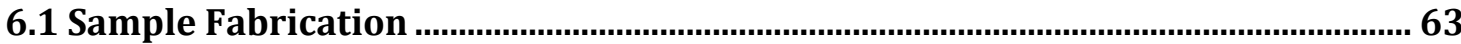

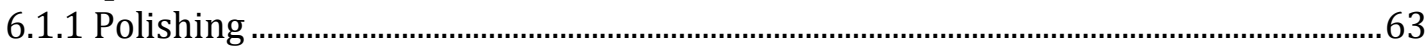

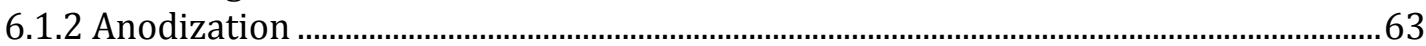

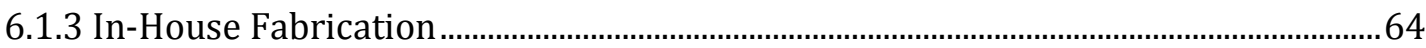

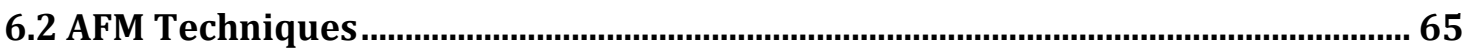

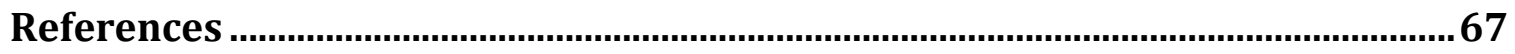




\section{LIST OF TABLES}

Table I. Electrochemical series for selected metals (Jacobs, Gilbert and Urban)

Table II. Summary of testing conditions for IV-AFM and C-AFM experiments

Table III. One-way ANOVA of surface roughness post anodization .26

Table IV. One-way ANOVA of surface roughness pre- anodization... 27

Table V. One-way ANOVA on (1Hz) IV-AFM measured scratch depth 38

Table VI. One-way ANOVA on C-AFM (B) average measured current........ 47 


\section{LIST OF FIGURES}

Figure 1.1 Elastic modulus of common biomedical alloys in comparison to cortical bone. 3

Figure 1.2 Diagram of metal M dissolution where metal ions $M^{n+}$ are released into solution and metal electrons are consumed by the reduction of $\mathrm{H}^{+}$to $\mathrm{H}_{2}$.

Figure 1.3 Beam bounce concept of how an AFM works to record topographical and force data as the AFM tip is translated across a substrate. 16

Figure 1.4 Surface manipulations on a polycarbonate substrate using AFM nanolithography software and Olympus AC 160TS cantilever (10 um scan).

Figure 2.1 Experimental scratch simulation showing how the Asylum Research MFP-3D AFM and MicroAngelo ${ }^{T M}$ lithography software was used to create scratches on Ti-6Al-4V samples for both in-vitro AFM and CAFM experiments.

Figure 2.2 Microstructure of Ti-6Al-4V received from Beaumont Hospital, with light alpha grains bordered by dark beta regions. (200x) (Image courtesy of Katie Miyashiro).......

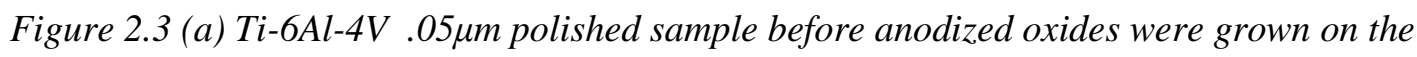
surface. (b) Ti-6Al-4V sample after anodization, where the golden yellow hue is representative of the change in the index of refraction caused by the growth of the oxide thickness.

Figure 2.4 In-vitro Petri dish BioHeater fluid testing cell used for IV-AFM nano-repassivation experiments. (Image courtesy of Asylum Research).

Figure 2.5 Schematic of the Petri Dish Heater assembly (top). Where the heater plate easily fits on the MFP-3D scanner and is held in place with a magnetic dish clamp.

(Courtesy of Asylum Research)

Figure 2.6 Temperature versus time plot of Bioheater sample holder during IV-AFM experiments indicating that sample and testing conditions were held constant throughout testing. 
Figure 2.7 Expected observation of repassivation after scratching is created with the AFM. Showing that overtime repassivation is observed as the z-sensor data changes.

Figure 2.8 Expected observations of conductance measurements after scratch is created with the AFM. The drop in conductance overtime represents the observation of repassivation around the created scratch.

Figure 2.9 C-AFM AFM (10 um scan) current contrast image of current measurements overlaid on a z-sensor topography scan. Image clearly indicates current detection around scratch.

Figure 2.10 C-AFM (A) average temperature profile of experiments, showing lower testing temperatures than the desired target testing temperatures.

Figure 3.1 (a) AFM image of a nano scratch created in the $1 \mathrm{~Hz} I V-A F M(b)$ AFM image of a scratch created in the $3 \mathrm{~Hz} I V-A F M$ experiment, where the color bar on the right shows a depth profile of approximately $30 \mathrm{~nm}$ for both experiments (Both images are $10 \mu \mathrm{m}$ scans)

Figure 3.2 IV-AFM $(1 \mathrm{~Hz})$ in-vitro max scratch depth that was monitored over time showing variance in scratch depth between experiments performed in $\mathrm{HBSS}$ at $37^{\circ} \mathrm{C}$.

Figure 3.3 IV-AFM (3Hz) in-vitro scratch depth monitored over time graph showing small changes in scratch depth throughout the experiments performed in HBSS and controlled with the AFM at 37 C..

Figure $3.4($ a)C-AFM (A) z-sensor profile image with current measurements superimposed on scanned topography. (b) Same image as (a), but only showing z-sensor depth of scratch with color bar showing scratch depth (10 $\mu$ m AFM scan).

Figure 3.5 C-AFM (A) experiments performed with $3.63 \mathrm{~V}$ bias at $20 \mathrm{C}$. (a) Is the average current measurements over time after AFM nano-scratches were created on Ti-6Al-4V substrates and $(b)$ is the amount of variance between replicated experiments. Measured slope is rate of decreasing current and error bar are standard deviation. 
Figure 3.6 C-AFM (A) experiments performed with $3.63 \mathrm{~V}$ bias at $37 \mathrm{C}$. (a) Is the average current measurements over time after AFM nano-scratches were created on Ti-6Al-4V substrates and $(b)$ is the amount of variance between replicated experiments. The measured slope is the rate of decreasing current and error bar are standard deviation. 43 Figure 3.7 C-AFM (A) experiments performed with $3.63 \mathrm{~V}$ bias at $45 C$. (a) Is the average current measurements over time after AFM nano-scratches were created on Ti-6Al-4V substrates and $(b)$ is the amount of variance between replicated experiments. Measured slope is rate of decreasing current and error bar are standard deviation. 44

Figure 3. 8 C-AFM (A) bar chart showing an increase in overall current detection with an increase in testing temperature. 45

Figure 3.9 Range of average current measured in $C-A F M(B)$ experiments over time after AFM manipulation of Ti-6Al-4Vsubstrates with a 3.63V bias applied at $20 \mathrm{C}$. 46

Figure 3. 10 (a) AFM current measurements in C-AFM C in HBSS at $37 \mathrm{C}$ and (b) Current

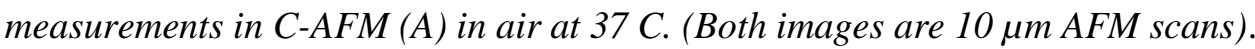
48

Figure 3. 11 AFM cantilever images comparing pre and post experiments. (a) is a image of an unused cantilever at $8335 x .(b)$ is the same cantilevers post experiments at 11,581 $x$ showing evidence of tip damage during experiments as well as debris left on the tip.

Figure 4. 1 Graph of estimated current densities observed in C-AFM (A) experiments 52 Figure 4. 2 Conductivity plotted over time showing a decreasing conductance and representing the observation of repassivation in $C$-AFM (A) performed in air at corresponding temperatures.

Figure 4. 3 Graph of electron mobility, using the current data in C-AFM (A) experiments showing a reduction in electron mobility as repassivation occurs. 54 Figure 4.4 (a)Conductivity measurements at 20, 37, and 45 C showing as temperature 
increase so does conductivity and (b) shows the best function of the plotted data used to calculate the $E_{g}$ values. 


\section{INTRODUCTION}

Metallic alloys have many uses in orthopaedic devices. Of these alloys used in devices, the most commonly found metals are stainless steel, cobalt chromium, and titanium alloys. The success of these alloys is due to their inherent ability to resist degradation in-vivo as well as minimize host immunological foreign body responses (FBR). This natural ability of corrosion resistance and biocompatibility is primarily due to the alloys capacity to form a protective inert oxide layer that prevents them from causing adverse reactions and further oxidizing. The excellent biological inertness and corrosion resistance ability are direct consequences of the chemical stability and structural integrity of the oxide layer. This ability of an oxide to self-heal (repassivate) is also critical in long term changing environments due to the dynamic nature of orthopaedic implants continuously being exposed to forces during joint articulation. With such demanding environments, late on-set infections have emerged as a relatively new problem associated with the corrosion of orthopaedic implants. To date, the study of passivation and repassivation has mostly neglected the effects of implant corrosion and its immunological FBR's to cause late on-set infections. Therefore, since this passive layer plays such a vital role in the biocompatibility of an implant, it is of critical importance to understand the ability of this film to passivate and repassivate in-vivo.

In an effort to further define the problem of late on-set infections, this thesis will focus on assessing in-vitro nano-repassivation of Ti-6Al-4V. Providing a framework for future research on the poorly defined problem of late on-set infections related to implant corrosion. Through in-vitro atomic force microscopy (AFM) scratch test methods, the development of experiments that more closely emulate conditions of in-vivo joint articulation were performed. The AFM was used to create nano-scratches using AFM lithography techniques. Simultaneously, the AFM was also used to characterize the changes in current measurements over time to capture the kinetics of in- 
vitro repassivation. The characterizations of thermal effects of oxide growth and electron mobility were also evaluated for possible effects on repassivation.

\subsection{Metallic Orthopaedic Implants}

Orthopaedic medical devices over the years have restored mobility, reduced pain and have improved the quality of life for many people with musculoskeletal diseases and orthopaedic trauma. The majority of orthopaedic devices are used in total joint arthroplasty (TJA) and fracture fixation devices based on global sales of all devices in 2000 (Long, 2008). TJA devices are most commonly used for implants of the hip, knee, ankle, shoulder, elbow, wrist, and fingers. Fracture fixation devices include a variety of devices and hardware components that include: spinal fixation devices, plates, wires, pins, screws, as well as other reconstructive devices.

For TJA and fixation devices the primary materials of choice are often metals. Metals are attractive in TJA and fixation devices due to properties such as: biocompatibility, low cost, manufacturability, high elastic modulus, tensile strength, ductility, and corrosion resistance (Wang, Currier, \& Yaszemski, 2006). Of the metals readily available for orthopaedics, ASTM F1472 Ti-6Al-4V has been identified to be superior to other alloys because its elastic modulus (Figure 1.1) is most similar to the replaced biological load bearing structures like cortical bone. This paper focuses on ASTM F 1472 Ti-6Al-4V using AFM techniques to study the properties of corrosion and the kinetics of nano-repassivation. 


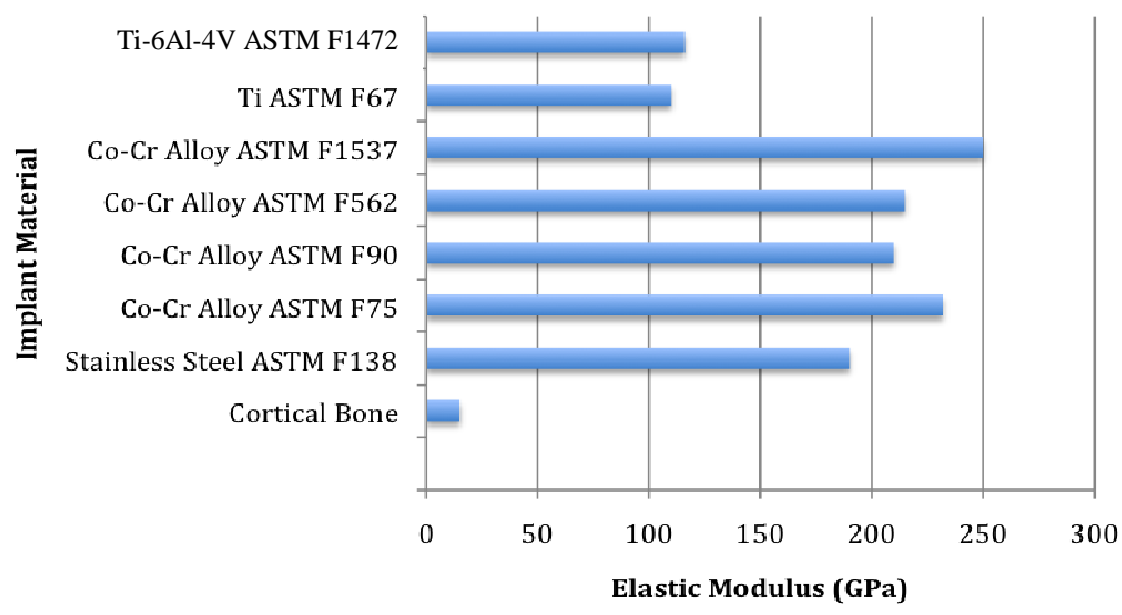

Figure 1.1 Elastic modulus of common biomedical alloys in comparison to cortical bone.

\subsection{Orthopaedic Infections}

There are many factors that can be associated with orthopaedic infections. In general, orthopaedic infections are classified into three stages: early (less than two weeks), delayed (2-10 weeks), and late (more than 10 weeks) infections (Trampuz \& Zimmerli, 2006). The majority of orthopaedic surgical procedures are often invasive and create large areas of trauma during the placement of the implant. The large open wounds allow for the possibility of microbial contamination as well as biofilm adherence to implant surfaces that can lead to infections. These biomaterial-associated infections are difficult to cure with antibiotics; in many cases replacement of the implant is the only remedy (Van de Belt, Nuet, Schenk, Van Horn, Van der Mei, \& Busscher, 2001). After the device is implanted within the host it is recognized as a foreign body where immunological FBR's cause macrophages to adhere to the surface (Mu, Kobayashi, Sumita, Yamamoto, \& Hanawa, 2000). If after a period of time the host continues to reject the implant with FBR's, adverse biological reactions can result in infections and implant failure (Jacobs, Gilbert, \& Urban, 1998).

In addition to the triggering of FBR's, the presence of corrosion has also been shown to cause late on-set infections. Corrosion of an implant is caused by localized mechanical or chemical 
stresses that inherently remove a protective oxide layer that all orthopaedic implants rely on. With the removal of this protective oxide layer the metal becomes reactive with its environment resulting in continued corrosion and the triggering of immunological FBR's. If corrosion continues, the localized effects can lead to the lowering of $\mathrm{pH}$ and the increase in temperature that are conducive to infection as well as the rate in which corrosion will occur.

\subsubsection{Microbial Contamination}

The pathogenesis of infection is related to microbial contamination that can grow in biofilms known as glycocalix, making it difficult to eliminate all adsorbed microbes (Khan, Rehman, Ali, Sultan, \& Sultan, 2008), (Gristina \& Costerton, 1985). On these biofilms, micro-ecosystems allow microbial strains to cultivate and adhere to implants during implantation or revision surgeries (Van de Belt, Nuet, Schenk, Van Horn, Van der Mei, \& Busscher, 2001). Once the microbe has established itself on an implant it is often difficult to remove as the glycocalix film acts as a protective layer that prevents the microbe from host immune responses and environmental attacks (Costerton, et al., 1987). With the presence of these biofilms, in many cases postoperative urogenital tract infections or dental treatments can introduce microorganisms into the host. Once in the host they can be transported by the blood stream where they can find their way to implant sites incubating biofilms and resulting in late on-set hematogenous infections (Stinchfield, Bigliani, Neu, Goss, \& et al. 1980),(Van de Belt, Nuet, Schenk, Van Horn, Van der Mei, \& Busscher, 2001). The development of these infections is influenced by many factors relating to the following: type of operation, operating room environment, and pathogen type. The pathogens that are predominately found in orthopaedic infections are staphylococci epidermidis and aureus, propioibacterium, anaerobic peptococci, and streptococci (Lecuire, Gontier, Carrere, \& al., 2003),(Tezer, Kuzgun, Hamzaoglu, Ozturk, Kabukcuoglu, \& Sirvanci, 2005),(Soultanis, 
Mantelos, Pagiatakis, \& Soucacos, 2003),(Van de Belt, Nuet, Schenk, Van Horn, Van der Mei, \& Busscher, 2001).

\subsubsection{Foreign Body Responses (FBR's)}

The foreign body reactions begin as wound healing, including the accumulation of fluid at the site of injury, infiltration of inflammatory cells to debride the area, and the formation of granulation tissue. However, the persistent presence of a biomedical implant or microbial contaminant will inhibit full healing. Even titanium, a commonly used biocompatible material, is considered a foreign body to which a host response is inevitable to some degree. Therefore, rather than the resorption and reconstruction that occurs in wound healing, the foreign body reaction is characterized by the release of macrophages, formation of foreign body giant cells, encapsulation of the foreign object, along with continued chronic inflammation at the surface interface of the material (Mu, Kobayashi, Sumita, Yamamoto, \& Hanawa, 2000). The detailed sequences of cellular events are beyond the scope of this paper. Therefore, a basic description of the cellular events include: protein adsorption, monocyte/macrophage adhesion, and macrophage fusion into foreign body giant cells. These interactions between the biomaterial, and the cellular communication amongst the present cells around the implant, dictate the overall host response and outcome of the patient (Anderson, Rodriguez, \& Chang, 2008).

FBR's can be influenced by ions being released due to corrosion of an implant. In a study performed by Mu et al. (2000), it was discovered that active oxygen species generated by macrophages are clearly a cause of ion release from the corrosion of titanium. With the accumulation of these macrophages a "respiratory burst" is created where the local environment surrounding the implant is saturated with oxygen species of the form of $\mathrm{H}_{2} \mathrm{O}_{2}$. This species is highly oxidative thereby increasing the rate of release of ions $\mathrm{Mu}$, Kobayashi, Sumita, Yamamoto, \& Hanawa, 2000). With the presence of corrosion by-products in the body it is also 
known that proteins can affect the corrosion behavior of metals and can either inhibit or accelerate corrosion (Khan, Williams, \& Williams, 1999),(Mu, Kobayashi, Sumita, Yamamoto, \& Hanawa, 2000). Just as the protective oxide layer provides kinetic barrier, the adherence of proteins will also contribute to this barrier and contribute to the inhibition of corrosion.

\subsubsection{Late On-Set Orthopaedic Infections}

For orthopaedics, late on-set infections can result in greater surgical problems. Since implant removal is not always easy, it can cause patients to experience severe pain and discomfort (Van de Belt, Nuet, Schenk, Van Horn, Van der Mei, \& Busscher, 2001). In 1993, the overall annual cost of orthopaedic implants associated with infection ranged between $\$ 150$ and \$200 million dollars in the US (Sculo, 1993). In the past, many of these late on-set infections were commonly associated with microbial contamination or intra-operative seeding. Today, despite the advancement of intra-operative antibiotic prophylactics, strict hygienic protocols, and special sterile enclosures, orthopaedic surgery infection rates still range from $1 \%$ to $3 \%$ (Antti-Poika, Jofefsson, Konttinen, Lidgren, et.al, 1990). It has also been shown that as devices become more complicated such as with spinal arthrodesis treatments, postoperative infection problems increase to an $8.5 \%$ infection rate (Soultanis, Mantelos, Pagiatakis, \& Soucacos, 2003). These increased rates can be associated to the device having multiple fasteners and multiple surface interactions making it susceptible to fretting corrosion.

With the presence of metal fretting corrosion at device interfaces along with local periimplant foreign body-type granulomatous (giant body cell) reactions sterile abscess and sinus formation can occur and later supply the environment for microbial growth (Gaine, Andrew, Chadwick, Cooke, \& Williamson, 2001). These localized corrosive environments, along with cellular responses is believed to be the cause of multiple cases of late on-set infections. Through the incubation of microorganisms the development of late deep wound infections is a more 
common occurrence resulting in patient discomfort, pain, and even paraparesis (Tezer, Kuzgun, Hamzaoglu, Ozturk, Kabukcuoglu, \& Sirvanci, 2005).

Lastly, it has been shown that the potential of microbial seeding during surgical procedures, implant corrosion, adherence of biofilms, or any combination of these conditions have shown to contribute to late on-set infections 1-7 years postoperatively (Akazawa, Minami, Takahashi, Kotani, Hanawa, \& Moriya, 2005);(Gaine, Andrew, Chadwick, Cooke, \& Williamson, 2001),(Tezer, Kuzgun, Hamzaoglu, Ozturk, Kabukcuoglu, \& Sirvanci, 2005);(Soultanis, Mantelos, Pagiatakis, \& Soucacos, 2003) (Jacobs, Gilbert, \& Urban, 1998),(Hallab, Merritt, \& Jacobs, 2001).

\subsection{Metallic Orthopaedic Corrosion Overview}

Metals, aside from all their successes and benefits, are still susceptible to adverse effects. Of these risks, corrosion of metallic implants is a major concern. In general, corrosion is the chemical or electrochemical reaction between a metal and its environment that results in the deterioration of a metal and its properties (Jones, 1996). The corrosion of orthopaedic devices is a complex multifactorial phenomenon that is affected by solution-chemistry, metallurgical, geometric, and mechanical parameters. In general, there are two main solution-chemistry factors that determine how and why a metal corrodes. The first factor is the thermodynamic driving force, also known as Gibbs free energy. The second factor that affects the solution chemistry is the kinetic barriers that can limit the rate of the corrosion reactions. Overall, these degradation processes can release degradation by-products that cause adverse biological reactions to the host (Jacobs, Gilbert, \& Urban, 1998). 


\subsubsection{Electrochemistry of Corrosion}

The fundamental reactions of corrosion are the electrochemical processes that involve the transfer of electrons from a metal to its surrounding environment. These reactions are commonly referred to as oxidation and reduction reactions. In the oxidation reaction, electrons are created and can be represented with reaction (1) where $\mathrm{M}$ represents a metal species with $n$ valence electrons, and $e^{-}$indicates a free electron (Temenoff \& Mikos, 2008).

$$
M \rightarrow M^{n+}+n e^{-}
$$

In a corrosion cell or corrosive environment the site on the metal where oxidation occurs is called the anode and is also referred to as the anodic reaction in the corrosion cell. Conversely to the oxidation site, the location where reduction reactions occur is referred to the cathodic reaction. The cathodic reactions are dependent on the environment that they are subject to. For example if the environment is acidic as can be the case around orthopaedic devices where inflammatory responses are present then, $\mathrm{H}^{+}$ions are reduced to form hydrogen gas:

$$
2 \mathrm{H}+2 e^{-} \rightarrow \mathrm{H}_{2}
$$

In addition to the acidic environment, the human internal environment is rich in dissolved oxygen, which can also result in cathodic reactions where oxygen is reduced in acidic (3) or neutral environments (Temenoff \& Mikos, 2008):

$$
\begin{aligned}
& \mathrm{O}_{2}+4 \mathrm{H}^{+}+4 e^{-} \rightarrow 2 \mathrm{H}_{2} \mathrm{O} \\
& \mathrm{O}_{2}+2 \mathrm{H}_{2} \mathrm{O}+4 e^{-} \rightarrow 4\left(\mathrm{OH}^{-}\right)
\end{aligned}
$$

In orthopaedic corrosion, the combination of the anodic and cathodic reactions cause the metal to react as in reaction (1) where the metal is dissolved by giving up electrons. These electrons then migrate to the materials surface and react with the $\mathrm{H}^{+}$in the environment to form $\mathrm{H}_{2}$ through reaction (2) (Figure 1.2). With a decrease in $\mathrm{pH}$, which commonly occurs due to infection, the presence of $\mathrm{H}^{+}$is increased where increased rates of corrosion can take place. 


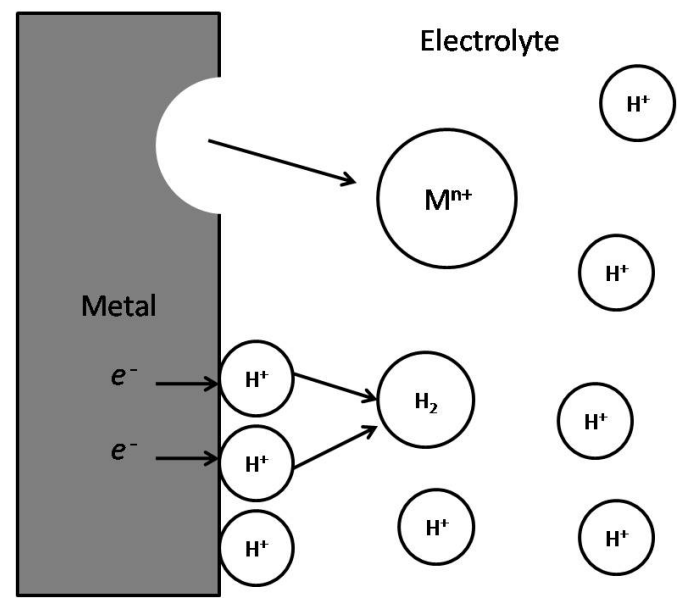

Figure 1.2 Diagram of metal M dissolution where metal ions $M^{n+}$ are released into solution and metal electrons are consumed by the reduction of $\mathrm{H}^{+}$to $\mathrm{H}_{2}$.

In a corrosion cell, in order for ions and electrons to readily move throughout these reactions, a fluid or electrolyte is required to carry these ions $(\mathrm{Mn}+$ and $\mathrm{H}+)$ allowing charge transfer to occur. Once these free ions are released from the metal during anodic reactions, the ions can migrate from the metal surface and can lead to the formation of metal oxides, metal chlorides, organometallic compounds or other chemical species (Figure 1.2) (Jacobs, Gilbert, \& Urban, 1998).

\subsubsection{Thermodynamic Driving Force of Corrosion}

The thermodynamic driving force known as the Gibbs free energy $(\Delta \mathrm{G})$ can be calculated with Equation 1.1 where $\Delta \mathrm{G}_{\text {red }}$ is the free energy for the reduction reaction, $\Delta \mathrm{G}^{\circ}$ is the standard state free energy (calculated at $25{ }^{\circ} \mathrm{C}$ with $.1 \mathrm{MPa}$ partial pressure of all gases used and $1 \mathrm{M}$ aqueous solutions), $\mathrm{R}$ is the universal gas constant, $\mathrm{T}$ is absolute temperature, and the bracketed values are the concentrations of the species involved in the reaction (Jacobs, Gilbert, \& Urban, 1998).

During fretting corrosion the concentration of species is increased, which can result in increased corrosion activity.

$$
\Delta \mathrm{G}_{\mathrm{red}}=\Delta \mathrm{G}^{\circ}+\mathrm{RT} \ln \left([\mathrm{M}] /\left[\mathrm{M}^{\mathrm{n}+}\right]\left[e^{-}\right]\right)
$$


$(\Delta \mathrm{G})$ provides the energy needed for corrosion (oxidation and reduction reactions) to occur. For example, if the $\Delta \mathrm{G}$ for a metal is negative then oxidation occurs spontaneously and the metal will begin to corrode. If the $\Delta \mathrm{G}$ is positive then the reactions become reductive and metal begins to gain electrons. When the $\Delta \mathrm{G}$ is zero, this is the state in which the metal is in an equilibrium state and no corrosion occurs. Along with the free energy, there is an electric potential that is created across the metal-solution interface. This potential is a measure of the reactivity of the metal and can be used to determine the amount of driving force for metal oxidation to occur. Standard state electrode potentials such as Table I, show the electrical potential of the reduction reactions a given material. For metals, the more negative the potential in a solution, the more reactive it will be (Jacobs, Gilbert, \& Urban, 1998). According to Table I. as electrical potential becomes more positive the metal becomes more noble indicating that there is little or no driving force for corrosion. Titanium on the other hand has a negative potential indicating that it would be susceptible to corrosion in an in-vivo environment. However, with such a high driving force its reactivity with an oxygen rich environment is the reason it is able to form a protective oxide layer.

Table I. Electrochemical series for selected metals (Jacobs, Gilbert and Urban)

$\begin{array}{ccccc} & & \text { Reaction } & & \text { Potential (V) } \\ \text { Noble } \quad \mathrm{Au}^{3+}+3 \mathrm{e}^{-} & \longleftrightarrow & \mathrm{Au} & 1.42 \\ \mathrm{O}_{2}+2 \mathrm{H}_{2} \mathrm{O}+4 \mathrm{e}^{-} & \longleftrightarrow & 1 / 2 \mathrm{H}_{2} & 0.00 \\ \mathrm{Ti}^{2+}+2 \mathrm{e}^{-} & \longleftrightarrow & \mathrm{Ti} & -1.60 \\ \mathrm{Mg}^{+}+\mathrm{e}^{-} & \longleftrightarrow & \mathrm{Mg} & -2.37 \\ & & & \\ & \mathrm{Na}^{+}+\mathrm{e}^{-} & & \mathrm{Na} & -2.71\end{array}$

\subsubsection{Passivation}

Passivation, or passivity of a metal, is caused by the formation of a thin, protective, hydrated oxide, corrosion-product surface film that acts as a barrier to the anodic dissolution reactions 
(Jones, 1996). Most alloys used in orthopaedics rely on these passive films to prevent oxidation from taking place (Jacobs, Gilbert, \& Urban, 1998),(Hallab, Jacobs, \& Katz, 2004). These passive films commonly referred to as kinetic barriers, physically prevent the transport of metallic ions and electrons across the metal-solution interface. This unique oxide property of these materials limits the electronic exchange between the electrolyte and the metal preventing charge transfer and corrosion from occurring. In comparison to materials such as low carbon steels, electron tunneling occurs between the surrounding electrolyte and metal through the oxide and therefore, continuously corrodes (Schultz \& Hassel, 2003). If titanium did not have such a high surface energy and a high affinity to readily react with oxygen to form a passivation layer, it would react violently with the surrounding environmental chemical species until it reverted to its simplest ionic form (Jacobs, Gilbert, \& Urban, 1998). Thus, because of the role played by the passive oxide film of titanium it is important to consider any mechanical and tribological factors that can cause the oxide film to be abraded or fractured as oxide removal due to fretting and micro motion can result in corrosion (Jacobs, Gilbert, \& Urban, 1998),(Hallab, Jacobs, \& Katz, 2004).

\subsubsection{Repassivation (Macroscale)}

Repassivation is the active-passive corrosion behavior, which is the combination of active corrosion and passivation. Repassivation in orthopaedic applications can exist when stresses are applied to an existing passivated oxide film to the point where the amount of stress can expose the unoxidized base metal. At the instance that the oxide is removed a large driving force is created by the presence of a high negative electric potential between anodic exposed area and cathodic oxide regions on the surface. This large driving force causes the oxidation of the metal and corrosion to occur momentarily until the oxide layer is restored. According to Hallab et al. (2004) the extent and duration of the oxidation depends on the formation kinetics of the oxide film and is dependent on the metal species and corresponding electric potentials as noted in Table I. In an oxygen rich environment such as the human body it has been shown that Ti-6Al-4V alloy 
spontaneously forms a passive oxide layer that is predominately $\mathrm{TiO}_{2}$ and is approximately $2-7$ nm in thickness (Milosev, Hukovic, \& Strehblow, 2000). It has also been found that titanium repassivation layers tend to be different from the native oxide layer; due to the incorporation of ions within the surrounding environment (Geetha, Singh, Asokamani, \& Gogia, 2009). The oxide films react with the electrolyte and are found to undergo partial dissolution and reprecipitation causing the oxide composition to change with its environment (Takao, 2002). If this process of repassivation occurs in a cyclic pattern, where the metal alternates between active and passive corrosion, then the process is known as fretting corrosion. Fretting, is commonly found in all load bearing orthopaedic applications due to their multiple surface-to-surface interface interactions. These interfaces are susceptible to micro motion and over time can develop into fretting corrosion, where the generation of ions and wear debris can result. Once fretting becomes present, these foreign particles can find themselves in the local periprosthetic tissue where it is known to be of clinical concern to cause FBR's and infections (Hallab, Jacobs, \& Katz, 2004),(Hallab, Merritt, \& Jacobs, 2001),(Geetha, Singh, Asokamani, \& Gogia, 2009).

\subsubsection{Repassivation (Nanoscale)}

Nanoscale repassivation is related to the nanostructures of a material and their protective thin layers that create corrosion conditions at the nanoscale. At the nanoscale, corrosion can occur layer by layer via a step-flow mechanism and slow etching rates (Maurice \& Marcus, Corrosion at the Nanoscale, 2009). Nanoscale studies have also shown that passive films are crystalline, where in the past they were thought to be amorphous (Maurice, Talah, \& Marcus, 1994). This crystallinity plays a role in the substrate structure and surface hydroxylation on the crystalline structure (Schultz \& Hassel, 2003). Surface roughness in addition plays a large role in the affect of surface energy on the surface. As a materials surface becomes rougher, the available surface energy generally decreases. This decreases the surface energy at the surface and increases the 
hydrophobic properties of the surface. Another large contribution to the affects of repassivation at the nanoscale is surface charge and surrounding particle charges at the surface. At the nanoscale as size decreases from the macroscopic limit towards nanoparticles and nanoclusters, properties exhibit a continuous evolution related to the growing importance of the surface and shape of the nanoparticles (e.g. closed-packed, cubic, facetted, etc...) without qualitative change in the electronic structure (Brechignac, Houdy, \& Lahmani, 2006). These properties below a critical size become more likely to change in the bulk itself, thereby affecting the nanoparticle or cluster as a whole (Spiegelman, 2006). These affects are due to the nature of the atomic structure, which must now be taken into account explicitly and partly due to the quantum nature of the electrons and ions participating in the chemical reactions. In addition to these effect of surface charges changes in $\mathrm{pH}$ can create conditions that are acidic as commonly associated with in-vitro corrosion and infection. In this acidic condition an abundance of $\mathrm{H}^{+}$ions can contribute to the affects of nanoparticle and nanoclustering and overall affect the chemical reactions at the surface of a repassivating implant.

\subsubsection{Oxide Growth Models}

However, as mentioned earlier, repassivation has been studied in detail on the macro and microscales. Such studies have developed oxide film growth models that have been theorized to model the kinetics of repassivation. Models such as the Ambrose model states that; as freshly exposed metal surfaces begin to grow an oxide film, the area fraction covered by the oxide, $\Theta$ increases with time (Ambrose, 1983). Assuming that film thickness is constant, current associated with film growth can be written as

$$
i_{\text {film }}=\left[\delta \rho z F A_{o}\right] /[M W][d \Theta / d t]
$$


$\delta$ is oxide thickness, $\rho$ is density of the oxide, $z$ is charge per cation, $F$ is Faraday's constant, $A_{o}$ is scratch area, and MW is molecular weight of the oxide. This model was then modified by Gilbert et al. where it was predicted that the total repassivation current was broken up into two separate current paths that describe oxide film growth Equation (4.5) and ionic dissolution Equation (4.6).

$$
i_{\text {diss }}=j_{\text {crit }} A_{o}(1-\Theta) \exp \left[\eta_{d} / b_{a}\right]
$$

The model assumes Tafel-like behavior, where $b_{a}$ is the Tafel slope for dissolution, $\eta_{d}$ is the over potential for the dissolution reaction, $j_{c r i t}$ is critical current density for passivation, and (1- $\Theta$ ) is the area fraction of exposed metal. Therefore, the summation of Equation (1.2) \& (1.3) can be used to describe repassivation of an oxide film with measured peak currents, measured time constant for repassivation, known oxide film thickness, sample over potential, and exchange current density for a given ionic solution (Equation 1.4) (Goldberg \& Gilbert, 1998).

$$
i_{\text {tot }}=\left[\delta \rho z F A_{o}\right] /[M W][d \Theta / d t]+j_{c r i t} A_{o}(1-\Theta) \exp \left[\eta_{d} / b_{a}\right]
$$

\subsubsection{Previous Studies of Orthopaedic Repassivation}

The investigation of oxide fracture and repassivation of titanium has been performed in the past using novel scratch test methods while being submerged in a saline solution and potentiostatically held (Gilbert, Buckely, \& Lautenschlager, 1996). In these studies the authors were able to collect and estimate the current excursion and time constants for repassivation. During these experiments Gilbert et.al also considered the effects of the applied loads during oxide fracture, sample potentials, $\mathrm{pH}$ differences, and aeration during experiments. In their conclusions, it was observed that $\mathrm{pH}$ and applied potential had significant effects to higher open circuit potentials (OCP), which was also correlated to a higher repassivation rate. In a similar study repassivation was 
observed looking at OCP achieved during mechanical abrasion in buffer solutions and bovine serum with varying $\mathrm{pH}$ (Contu, Elsener, \& Bohni, 2004). Much like in the first study mentioned, the authors discovered that as acidity and mechanical abrasion load increased, OCP increased relating to an increase in repassivation rates. Experiments were also performed in a protienaceous bovine serum under physiological $\mathrm{pH}$ conditions. There it was found that repassivation was not affected by the presence of proteins, but did show that commercially pure titanium repassviated more quickly than Ti-6Al-4V based on the OCP and lower critical current densities were observed in each condition. However, the explanation of the higher repassivation rate in commercially pure titanium could not be explained and only suggested that epitaxy of the oxide layer was altered by the compositional differences in the microstructure.

In addition to observing repassivation through electromechanical experiments on the macro and micro scale, many studies have been performed investigating what types of oxides are reformed during repassivation. In one study electrochemical oxidation was performed on Ti-6Al-4V in physiological solutions where the samples were removed and the oxides (passivated films) were observed and characterized using X-ray photoelectron spectroscopy (XPS) and electrochemical impedance spectroscopy (EIS) (Milosev, Hukovic, \& Strehblow, 2000). The observations compared air-exposed oxides to the varied electrochemical oxidation conditions and observed that the oxides are primarily $\mathrm{TiO}_{2}$. It was also observed that amounts of $\mathrm{TiO}$ and $\mathrm{Ti}_{2} \mathrm{O}_{3}$ suboxides decreased as electrochemical potentials were increased. In addition to the decreased suboxides, as potentials increased it was observed that $\mathrm{Al}_{2} \mathrm{O}_{3}$ increased in the outer oxide layer.

Apart from the studies described here, there has been little published work to date examining repassivation and its effects on the nanoscale; however, the abundant investigations at the macro and micro scale do offer insight to in-vitro repassivation and corrosion. 


\subsection{Atomic Force Microscopy (AFM): Method to Investigate Nano-Repassivation}

Atomic force microscopy (AFM) is a form of scanning probe microscopy and uses the sense of touch with a cantilever to determine substrate characteristics. Similar to how a visually impaired person reads brail through the sensation of touch with their fingertips. The AFM probe contacts the specimen and is physically moves up and down along the substrate as the sample is traversed beneath the tip. The movement or deflection of the probe is then translated by bouncing a laser off a focused mirror onto the back of the surface of the probe and recorded using a four-quadrant photo detector. Figure 1.3 shows a more detailed general description of how the beam bounce concept works with the combined cantilever tip, laser, four-quadrant photo detector, z-piezo and an extremely accurate feedback loop to provide data and 3D images. The AFM has the potential of measuring small forces on a local scale, performing nano-lithography, conducting current measurements, performing surface characterization, and can be applied to simulated physiological environments to investigate biological samples (Asylum Research, 2009).

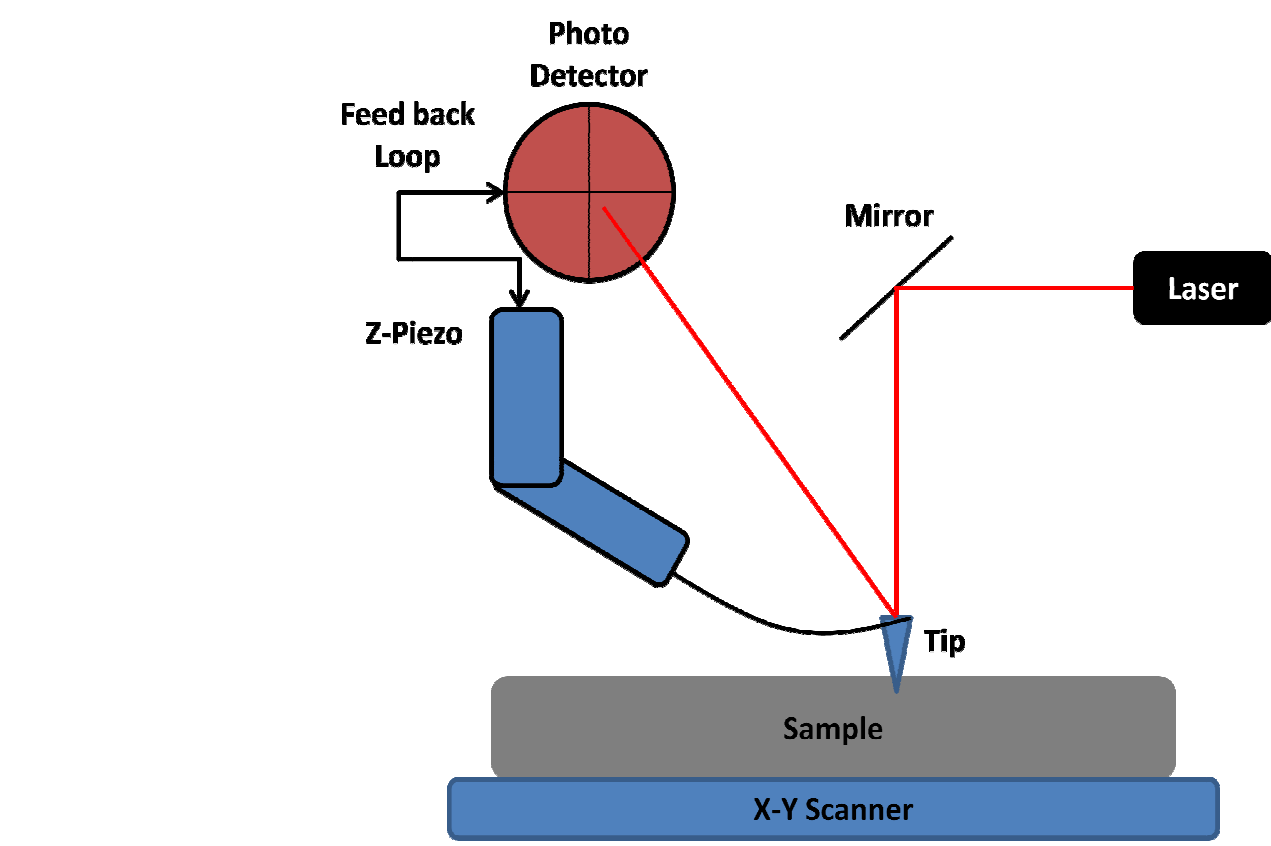

Figure 1.3 Beam bounce concept of how an AFM works to record topographical and force data as the AFM tip is translated across a substrate. 


\subsubsection{AFM Imaging Modes}

In general the AFM can operate in dynamic (AC-mode) and contact modes with sub nanometer resolution capabilities. With either mode the cantilever tip is deflected in response to the interaction forces between the tip and the substrate. The deflection is then quantified and translated using the beam bounce concept as mentioned previously. In contact mode the cantilever tip is set at a predetermined deflection and is continuously controlled as it rasters across the substrate. Dynamic mode, or AC mode, is a more delicate approach to imaging where the cantilever is oscillated at a frequency bouncing the cantilever across the substrate. In this mode, instead of setting a predetermined deflection, a set amplitude is held as the tip is rastered across the substrate.

\subsubsection{Nanolithography}

Lithography and manipulation capabilities have been around for quite some time on the microscale, especially in the microfabrication industry. It was in 1990 when IBM first used the scanning tunneling microscope (STM) to spell out IBM with manipulated Xe atoms (Eigler \& Schweizer, 1990). Today, AFM nanolithography is being utilized in applications including surface scratching, patterning (Figure 1.4), localized surface oxidation, nanotube particle, molecular manipulation and many other applications (Asylum Research, 2008). 


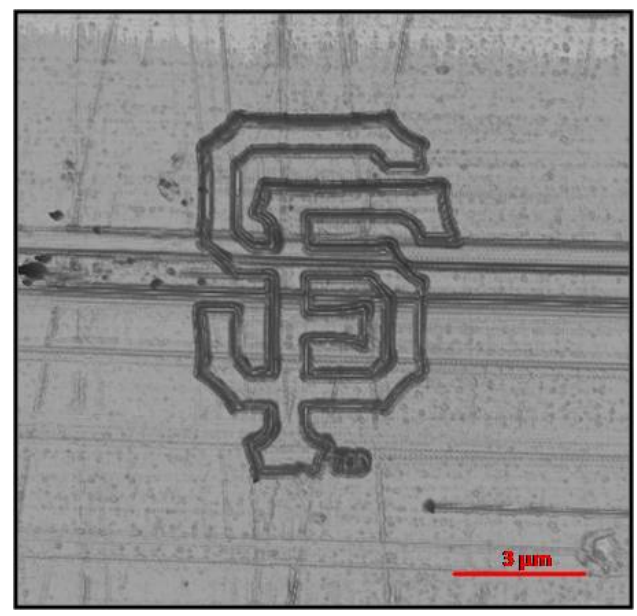

Figure 1.4 Surface manipulations on a polycarbonate substrate using AFM nanolithography software and Olympus AC 160TS cantilever (10 $\mu m$ scan).

AFM scratching and nano-patterning of silicon substrates has been a growing research topic in the microfabrication industry to scratch insulating layers using the tip, or micro-indenter, of an AFM (Santinacci, Zhang, \& Schmuki, 2005). Santinacci et al. (2005) used AFM techniques to create nano-scratches on Si oxide substrates using several loads. After patterning the silicon oxide, the scratches were refilled with a metal deposition process in attempts to create integrated circuits or nano wires. It was concluded that with AFM patterning, $50 \mathrm{~nm}$ groove geometries provided well-defined metallic structures on silicon surfaces within several hundred nm resolution (Santinacci, Zhang, \& Schmuki, 2005).

AFM nano-scratching and indentation has also been used to investigate the possibility of measuring ultrathin film mechanical properties. Authors Crozier et al. (2000) developed techniques to evaluate and measure film thickness, flow stresses, and stiffness of the films (Crozier, Yaralioglu, Degertekin, Adams, Minne, \& Quate, 2000).

Although useful, AFM nano-scratching was shown by Khurshudov et al. (1996) that the integrity AFM tips during scratching were susceptible to degradation during repeated scratching and imaging. With performed nano-scratches it was observed that scratch depth decreased with 
the number of cycles and increased material hardness, more tip wear, and wear rates were present (Khurshudov, Kato, \& Koide, 1996).

\subsubsection{Conductive AFM}

Conductive AFM is another SPM method that uses standard AFM techniques with a special cantilever holder. In this technique the AFM uses a transimpedance amplifier that allows for point contact electrical measurements using a metal coated or heavily doped tip. This tip serves as both a movable electrode, and force sensor allowing for useful material characterization applications. Conductive AFM allows for simultaneous mapping of the topography and current distribution of a sample with current measurements in the range of hundreds of femtoamps to nearly a microamp (Asylum Research, 2009). This capability has led to numerous studies of current-voltage characterization of many materials. Zorba et al. (2001) performed I-V measurements as a function of applied tip force on semiconductive pentace. Showing that steady electrical point contact between the conductive tip and sample provided reproducible I-V measurements as varying forces were applied (Zorba, Watkins, Yan, \& Gao, 2001). Similarly, I-V curves as a function of loading force on europium doped $\mathrm{ZnO}$ showed current measurements increased with increasing applied force (Asylum Research, 2009). Conductive AFM has been proven to be an indispensable technique to study morphological, electronic and material properties with high spatial resolution (Zorba, Watkins, Yan, \& Gao, 2001).

In addition to current measurements of conductive AFM systems, the use of conductive cantilevers and probes has led to production of stoichiometric oxides through local anodic oxidation with the tip (Cambel \& Soltys, 2007). Lazzarino et al. (2006) also created oxide structures in contact mode over gallium substrates where the substrate was grounded and a bias was applied through the tip. Conductive AFM has also been used to perform local oxidation and 
take measurements of faradic current or oxide reaction current (Yasushi, Yasuyuki, Genta, Tsutomu, \& Jun-ichi, 2007).

\subsubsection{AFM in Fluids}

The AFM is not limited to use in air; it can also be operated in liquids. The ability to function in fluids allows for the investigation of many surface-fluid interactions including those seen in biological samples. The use of fluids for most samples also eliminates the capillary forces that can affect the tip when imaging in air (Weisenhorn, Hansma, Albrecht, \& Quate, 1989). The fluid imaging capabilities of the AFM are also aided with the development of closed fluid cells and temperature controlled Petri dishes. These devises, such as the Asylum Research Petri Dish heater stage, allow for temperature control of biological samples within $0.1{ }^{\circ} \mathrm{C}$ and temperature ranges from $20{ }^{\circ} \mathrm{C}$ to $45^{\circ} \mathrm{C}$ (Asylum Reseach, 2008). The control of biological assemblies using AFM accessories has led to imaging and manipulation of biological samples at the nanoscale. Some examples include: the exploration of nano-manipulation of chromosomal DNA for genetic analysis, the disruption of antibody-antigen bonds, the dissection of biological membranes, nanodissection of protein complexes, and the controlled modulation of protein conformation (Fotiadis, Scheuring, Muller, Engel, \& Muller, 2002). Such studies have been valuable for many areas of research and with continued advancement can provide insight on other biological interactions.

\subsection{Project Objectives}

Recent findings on the frequency of late on-set infections occurring in orthopaedic patients 1-7 years postoperatively encouraged by implant corrosion requires further investigation of in-vivo repassivation to prevent delayed infections. Therefore, the goal of this research is to investigate AFM techniques to create simulated in-vitro nano-repassivation conditions as well as characterize 
repassivation on the nanoscale to better understanding in-vitro corrosion. The AFM techniques to be explored include:

1. Demonstrate the feasibility to create nano-repassivation conditions, as well as the ability to characterize in-vitro nano-repassivation via AFM techniques

2. Demonstrate the ability to characterize nano-repassivation in air, with the use of conductive AFM techniques

3. Demonstrate the ability to characterize in-vitro nano-repassivation with the use of conductive AFM techniques 


\section{MATERIALS AND METHODS}

\subsection{Experimental Overview}

ASTM F 1472 standard grade Ti-6Al-4V samples were divided equally into two different nano-repassivation scratch test experiments and identified as In-vitro AFM (IV-AFM) and conductive AFM (C-AFM) experiments. During testing all samples were kept separated in their designated sample holder boxes throughout the completion of the experiments. In all experiments the prepared oxide substrates were altered through means of nanolithography via an AFM cantilever tip in the pattern of a single line approximately $6 \mu \mathrm{m}$ in length (Figure 2.1). All samples were then characterized pre and post scratching via AFM techniques.

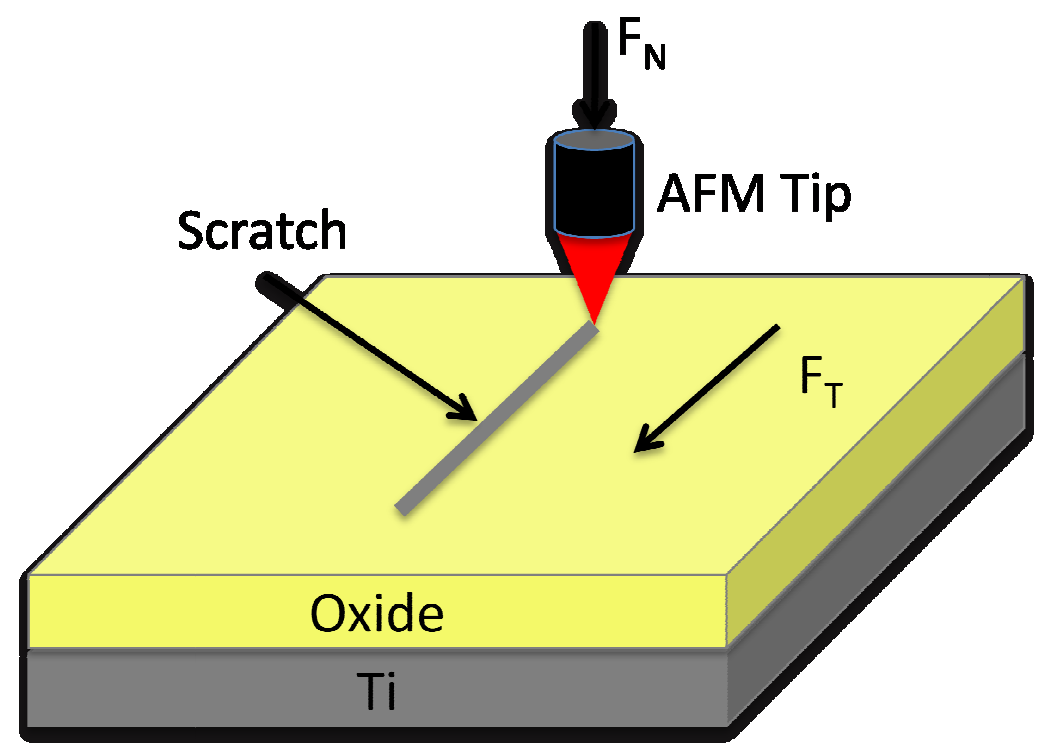

Figure 2.1 Experimental scratch simulation showing how the Asylum Research MFP-3D AFM and MicroAngelo ${ }^{T M}$ lithography software was used to create scratches on Ti-6Al-4V samples for both invitro AFM and CAFM experiments.

The data from the IV-AFM experiments allowed for the observations of repassivation on the nanoscale under in-vitro physiological conditions. With the characterization of the substrate using AFM topography (z-sensor) scans, effects of repassivation were observed. In the C-AFM experiments, a conductive AFM module was used to characterize electrical conductance to observe the effects of repassivation pre and post 
scratching in air at several different temperatures and in-vitro. Table II shows a summary of all the experiments performed and shows their differences and similarities between experiments.

Table II. Summary of testing conditions for IV-AFM and C-AFM experiments

\begin{tabular}{|c|c|c|c|c|c|}
\hline Scan Rate & $\begin{array}{c}\text { \# of } \\
\text { Experiments }\end{array}$ & Temp C & Sample \# & Cantilever & $\begin{array}{c}\text { Scan size } \\
\left(\boldsymbol{\mu m}^{2}\right)\end{array}$ \\
\hline IV-AFM & & & & & 10 \\
\hline $1 \mathrm{~Hz}(4.5 \mathrm{~min} / \mathrm{scan})$ & 3 & 37 & 1 & PPP-NCVH & 10 \\
\hline $3 \mathrm{~Hz}(1.4 \mathrm{~min} / \mathrm{scan})$ & 2 & 37 & 1 & PPP-NCVH & \\
\hline & & & & & \\
\hline C-AFM (A) (in-air) & & & & & 10 \\
\hline $2 \mathrm{~Hz}(2.13 \mathrm{~min} / \mathrm{scan})$ & 3 & 20 & 2 & CDT-NCHR & 10 \\
\hline $2 \mathrm{~Hz}$ & 3 & 37 & 3 & CDT-NCHR & 10 \\
\hline $2 \mathrm{~Hz}$ & 3 & 45 & 4 & & 10 \\
\hline & & & & & \\
\hline C-AFM (B) (in-air) & 3 & 20 & 5 & CDT-NCHR & 10 \\
\hline $2 \mathrm{~Hz}$ & 3 & 20 & 6 & CDT-NCHR & \\
\hline $2 \mathrm{~Hz}$ & 2 & 20 & 7 & & 10 \\
\hline $2 \mathrm{~Hz}$ & & & & & \\
\hline
\end{tabular}

\subsection{Materials}

Ti-6Al-4V material was donated by Beaumont Hospital Orthopaedic Research Center in Royal Oak Michigan. Before testing, the composition of the material was confirmed by energy dispersive X-ray spectroscopy (EDS) as 85.5 At\% Ti, 11.1 At\% Al, 3.4 At \% V and 89.7 Wt\% Ti, $6.5 \mathrm{Wt} \% \mathrm{Al}, 3.8 \mathrm{Wt} \% \mathrm{~V}$ which is in accordance to ASTM F1472 grade titanium alloy.

The Ti-6Al-4V used is a $\alpha-\beta$ titanium alloy. In this class of titanium, it contains an $\alpha$-stabilizing (aluminum) and $\beta$-stabilizing (vanadium) element. The overall structure is hexagonal closed packed (HCP), but the microstructure depends on the chemical composition, processing history, and thermal treatment. According to ASTM F 1472 this alloy is generally heat treated above 1000 ${ }^{\circ} \mathrm{C}$ to stabilize the $\beta$-phase and then quenched in oil to create the two phase structure. When quenched the microstructure becomes a martensitic $\alpha$-phase structure consisting if individual 
platelets which are heavily twinned and have an HCP structure that help give the alloy its strength. After quenching, the alloy is annealed allowing the $\beta$-phase to precipitate out of the $\alpha$ phase where it is then cold worked to further increase its strength. Figure 2.2 shows the microstructure of the as received alloy with the light colored plate like $\alpha$-phase and the dark intergranular $\beta$-phase around the edges.

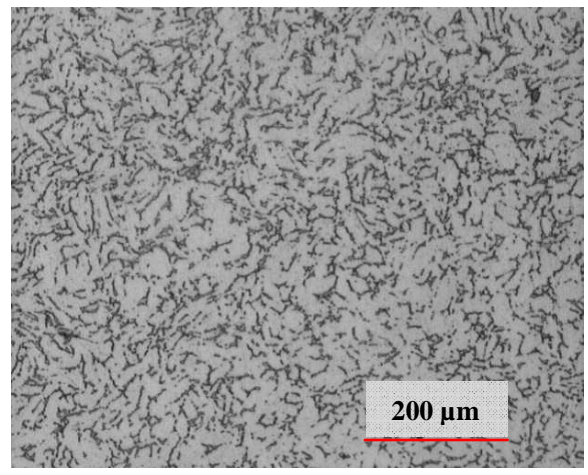

Figure 2.2 Microstructure of Ti-6Al-4V received from Beaumont Hospital, with light alpha grains bordered by dark beta regions. (200x) (Image courtesy of Katie Miyashiro)

\subsection{Sample Preparation}

\subsubsection{Polishing}

Ti-6Al-4V coupons were prepared in house into flat square samples approximately $10 \mathrm{~mm} \times 10$ mm x 2 mm dimensions (Figure 2.3) using a Leco V-50 precision diamond saw. All samples were then polished to a $.05 \mu \mathrm{m}$ surface finish sequential using standard metallography techniques. Coarse polishing was performed with the use of silicon carbide paper in progressive grits starting at 200 and finishing with 600. Fine polishing was achieved with the use of Buehler polishing wheels and pads and the use of sequential polishing media starting with $6 \mu \mathrm{m}$ diamond paste and finishing with $.05 \mu \mathrm{m}$ alumina suspension. After polishing all samples were characterized for surface roughness using an Asylum Research MFP-3D AFM in AC mode using a Nanosensors PPP-NCLR cantilever, where the average root mean squared (RMS) surface roughness was measured to be $3.90 \mathrm{~nm}$. 

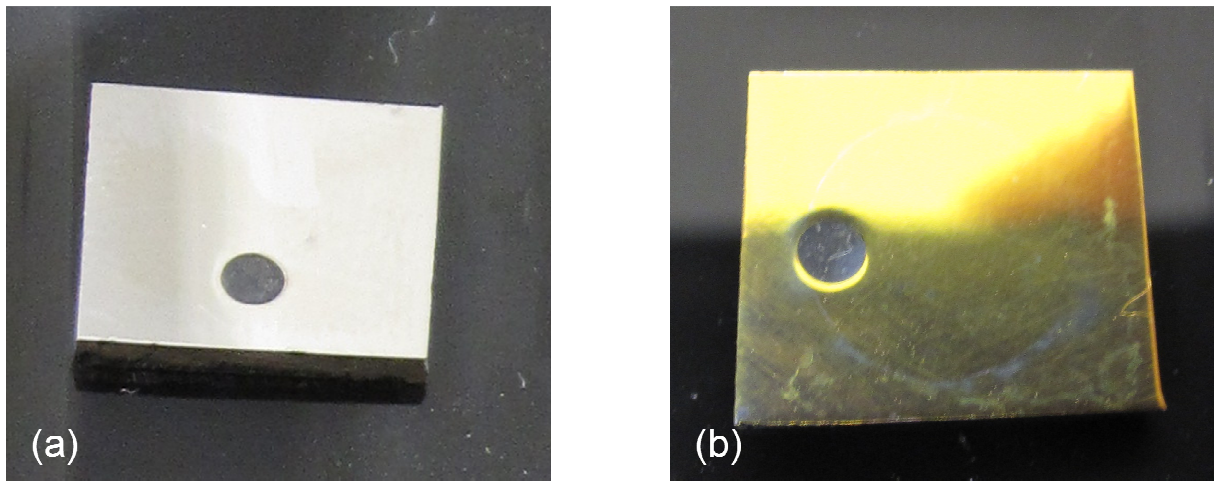

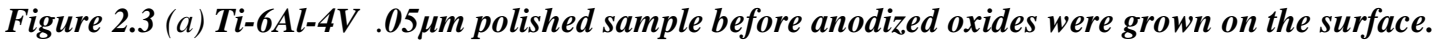
(b) Ti-6Al-4V sample after anodization, where the golden yellow hue is representative of the change in the index of refraction caused by the growth of the oxide thickness.

\subsubsection{Anodization}

After polishing, all samples went through an anodization process where oxide layers $\sim 15-20$ $\mathrm{nm}$ in thickness were grown. To grow the oxides, a Struers LectroPol-5 electropolisher was used to apply $10 \mathrm{~V}$ to the sample for 10 seconds in a $1 \mathrm{M}$ solution of $\mathrm{H}_{2} \mathrm{SO}_{4}$. The procedure was adopted from other research (Sul, Johansson, Jeong, \& Albrektsson, 2001), where the corresponding anodization parameters yielded a given oxide thickness based on voltage and time. Figure 2.3 shows the created sample with a golden yellow color after anodization which according to Sul et al. (2001) yielded 15-20 nm oxide layer. To ensure that an oxide was grown on all samples, a visual inspection, surface roughness, as well as dynamic secondary ion mas spectroscopy (D-SIMS) was performed to confirm altered oxide presence. D-SIMS was attempted to measure oxide thicknesses, but after unsuccessful attempts the data was only used for qualitative measurements of the altered oxide. In the visual inspection, all samples had the same golden yellow hue and according to Sul et al. (2001) corresponded to the change in the refractive index on the grown oxide layer. Post anodization RMS roughness was also measured on all samples at three random locations on the anodized surface. The averaged RMS roughness after anodization was $5.27 \mathrm{~nm}$ indicating an increase in roughness from the previous $3.90 \mathrm{~nm}$. This increase in surface roughness can be associated with the anodization process, where the added 
energy during the process permitted electron transfer and oxidation to occur on the surface through the existing oxide layer. However, there was aproximately $2 \mathrm{~nm}$ of variation which, is believed to be due to the methods of anodization and polishing (Sul, Johansson, Jeong, \& Albrektsson, 2001). One-way ANOVA of surface roughness also indicated that there were statistical differences between samples after anodization (Table III). With a $95 \%$ confidence interval $(\mathrm{CI})$ a P-value was calculated to be less than 0.05 . Therfore, indicating that the measured surface roughness between samples was a source of variation.

\section{Table III. One-way ANOVA of surface roughness post anodization}

\begin{tabular}{lcccccc} 
ANOVA & \multicolumn{7}{c}{ Source of Variation } & $S S$ & $d f$ & $M S$ & $F$ & P-value & F crit \\
\hline Between Groups & 20.64442 & 4 & 5.161105 & 9.2331 & 0.002167 & 3.47805 \\
Within Groups & 5.589786 & 10 & 0.558979 & & & \\
& & & & & & \\
Total & 26.23421 & 14 & & & & \\
\hline
\end{tabular}

Unfortunately, all samples were anodized one at a time due to the limited size of the electrochemical bath in the electropolisher used. As a result, the samples many factors could have affected the anodzation process. Factors such as; the concentration of the sulfuric acid between anodizing samples, the contact between electrode and sample, and the flatness of the sample from polishing are all possible reasons for the variation. One-way ANOVA comparison of the preanodized surface roughness also showed variability from sample to sample after polishing (Table IV). Although there was evidence of variation bewteen samples, the samples were still used for both the IV-AFM and C-AFM experiments. The variance is considered in the results as well as in the discussion sections. 
Table IV. One-way ANOVA of surface roughness pre- anodization

\begin{tabular}{|c|c|c|c|c|c|c|}
\hline Source of Variation & $S S$ & $d f$ & $M S$ & $F$ & $P$-value & F crit \\
\hline Between Groups & 12.91455 & 4 & 3.228638 & 5.039996 & 0.0174 & 3.47805 \\
\hline Within Groups & 6.406034 & 10 & 0.640603 & & & \\
\hline Total & 19.32059 & 14 & & & & \\
\hline
\end{tabular}

\subsubsection{AFM Experimental Sample Preparation}

After samples went through polishing and anodizing all samples were cleaned and dried prior to AFM experiments. The cleaning process involved 10 minute ultrasonic cleaning in deionized water (DI), followed by an additional 10 minutes in 99\% Isopropanol (IPA). After ultrasonic cleaning the samples were air blown dry with compressed air and placed in an oven at $50{ }^{\circ} \mathrm{C}$ for 1 hour. Once the drying process was complete the samples were placed in designated sample holders respectively for IV-AFM and C-AFM experiments.

\subsection{AFM Repassivation Test Methods}

\subsubsection{In-vitro AFM Experimental Setup}

The in-vitro testing cell was created with the use of the Asylum Research Petri-dish BioHeater accessory and allowed for controllable physiological testing conditions (Figure 2.4). In order to investigate in-vitro nanoscale repassivation of Ti-6Al-4V, AFM nano-scratch tests were developed and performed to observe the ability of Ti-6Al-4V to repassivate on the nanoscale. 


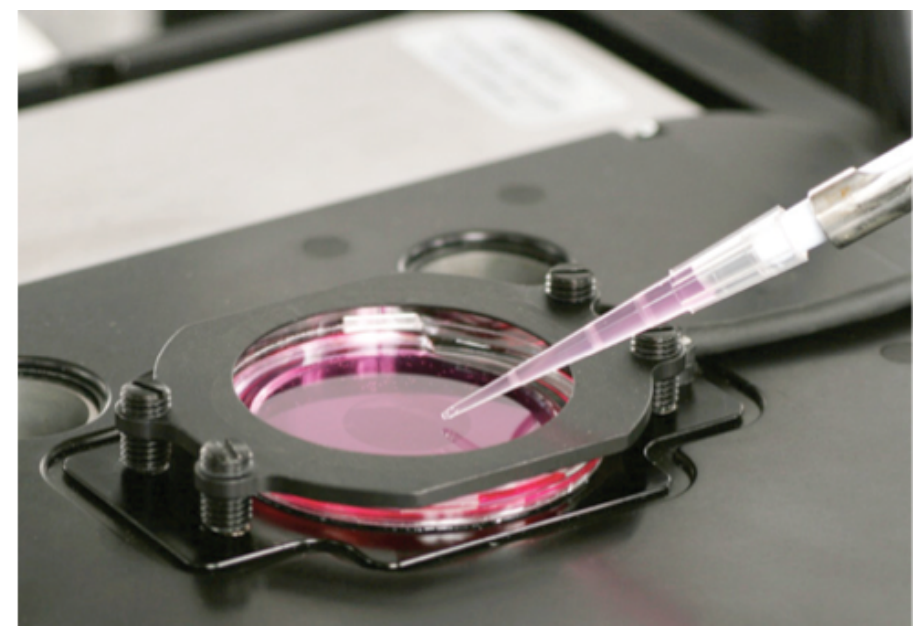

Figure 2.4 In-vitro Petri dish BioHeater fluid testing cell used for IV-AFM nano-repassivation experiments. (Image courtesy of Asylum Research)

Using an Asylum Research MFP-3D AFM in conjunction with the Petri-dish BioHeater, MicroAngelo $^{\mathrm{TM}}$ nanolithography software, NANOSENSORS ${ }^{\mathrm{TM}}$ PPP-NCVH silicon cantilevers, AFM lithography, and fluid imaging techniques; nano-scratches were created under simulated invitro repassivation conditions. The characterization of repassivation was monitored with the AFM z-sensor topography scans to measure scratch depth while continually rastering a $10 \mathrm{x} 10 \mu \mathrm{m}$ area around the $6 \mu \mathrm{m}$ scratch created.

All IV-AFM tests were controlled at $37^{\circ} \mathrm{C}+/-.05^{\circ} \mathrm{C}$ while samples were submerged in Hanks buffered saline solution (HBSS). The HBSS was used to simulate physiological fluids during testing. HBSS is a phosphate buffered saline that has been researched to be physiologically comparable to human blood with a $\mathrm{pH}$ of 7.35 and containing $\mathrm{Ca}^{+}, \mathrm{Na}^{+}$, and $\mathrm{K}^{+}$ions (Milosev, Hukovic, \& Strehblow, 2000).

The entire testing cell (Figure 2.5) consisted of a Petri dish that held the sample, HBSS, and was secured with magnetic clamps that fit on the AFM controller. A silicone membrane was used to seal and protect the cantilever holder from moisture as well as protect the AFM head from excess moisture during testing. 


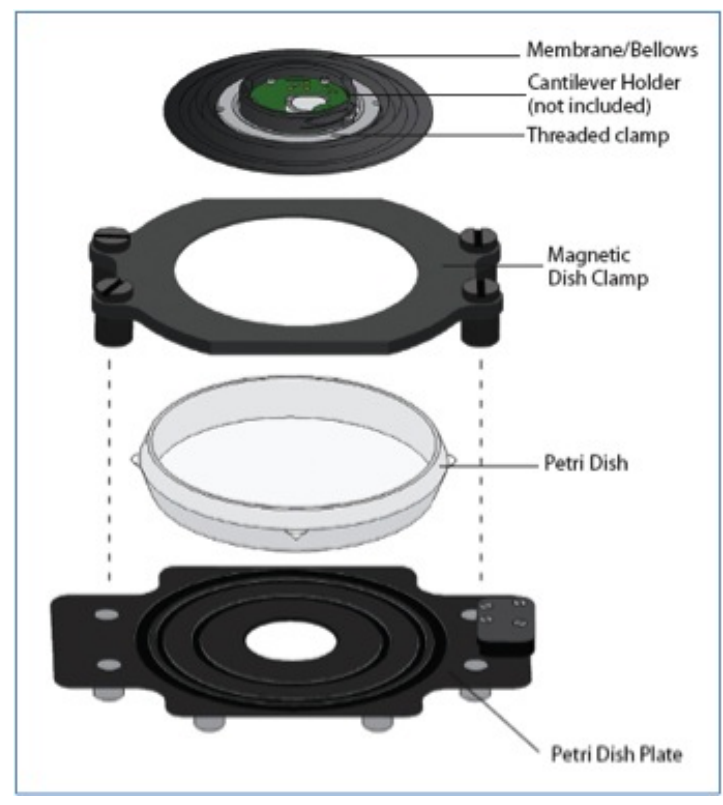

Figure 2.5 Schematic of the Petri Dish Heater assembly (top). Where the heater plate easily fits on the MFP-3D scanner and is held in place with a magnetic dish clamp. (Courtesy of Asylum Research)

\subsubsection{IV-AFM Repassivation Test}

IV-AFM experiments were performed in the materials engineering microscopy lab at California Polytechnic State University, San Luis Obispo. The IV-AFM experiments began with the fluid cell and sample being heated to testing conditions of $37^{\circ} \mathrm{C}$. While the fluid cell and sample were heated, a testing area $(10 \mu \mathrm{m} \times 10 \mu \mathrm{m})$ on the sample was located. Once a smooth, flat, and debris free area was located the entire system was allowed to equilibrate to $37^{\circ} \mathrm{C}$. The temperature was continuously monitored with the AFM MFP-3D software (Figure 2.6). After reaching equilibrium the experiments started with an AFM surface characterization in AC mode prior to performing nano-scratch. The characterization prior to scratching is regarded as the zero state of repassivation and provides the starting point to which each scan after scratching will be compared to. 


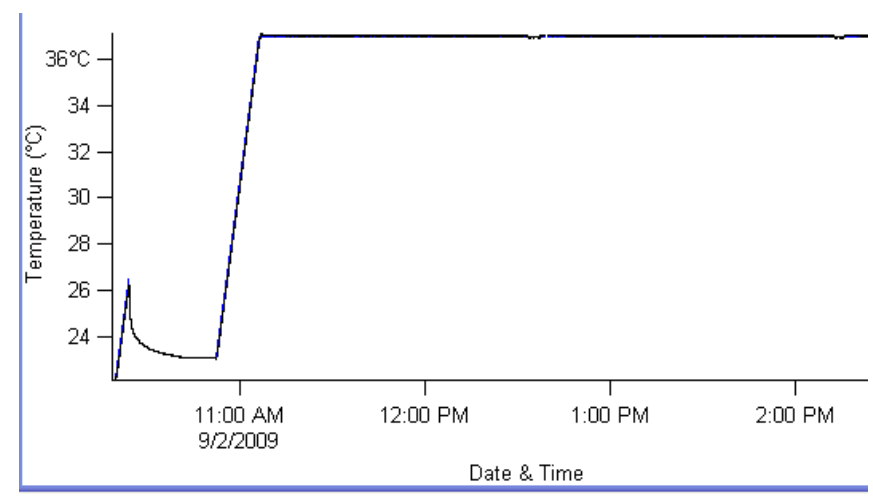

Figure 2.6 Temperature versus time plot of Bioheater sample holder during IV-AFM experiments indicating that sample and testing conditions were held constant throughout testing.

To perform the nano-scratch, the use of the MicroAngelo ${ }^{\mathrm{TM}}$ nanolithography software was used to control the silicon AFM cantilever to create a single $6 \mu \mathrm{m}$ scratch (Figure 2.1) The average applied force to create each scratch was $42 \mu \mathrm{N}$ and was dependent on the individual cantilever spring constants and surface morphology. Each scratch was performed at $125 \mathrm{~nm} / \mathrm{sec}$ and took approximately 48 seconds. After scratches were created with the AFM tip, the scratch was immediately characterized in contact mode at 3 and $1 \mathrm{~Hz}$ scan rates for 10 and 30 minutes respectively. The two different scan rates controlled the data acquisition with the control of the AFM raster rate. The higher the AFM rasters (increase in hertz) the faster the data was collected.

The data acquisition of the IV-AFM experiments were intended to capture the change in scratch depth over time using the z-sensor data collected over time with the AFM. Utilizing the AFM zsensor to characterize the surface after creating the nano-scratch would allow for the observation of changes during repassivation. Therefore, with the ability of the AFM to continuously monitor the created scratch depth with the z-sensor data, over time the observation of a decrease in maximum scratch depth would be representative of repassivation. Figure 2.7 is an example of an ideal plot of what the data should look like, showing continual changes in the z-sensor data and observing repassivation. By using the AFM to continually raster the scratched area, the topography (z-sensor) scans can be compared to any changes in the oxide substrate, which could 
then be correlated to repassivation or regeneration of the oxide layer. Changes in AFM z-sensor, such as illustrated in Figure 2.7, would demonstrate evidence of in-vitro repassivation on the nanoscale. IV-AFM was designed to demonstrate the ability to perform nano-scratches and characterize them in-vitro with the use of AFM z-sensor scans.

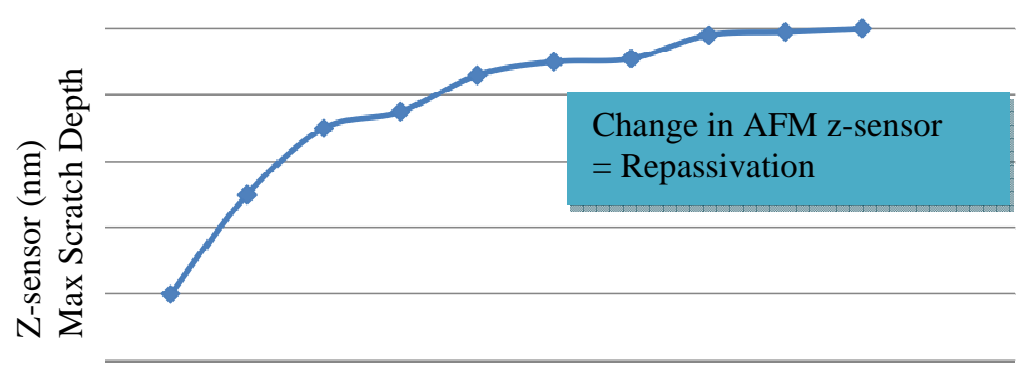

Time

Figure 2.7 Expected observation of repassivation after scratching is created with the AFM. Showing that overtime repassivation is observed as the z-sensor data changes.

\subsubsection{C-AFM Repassivation Test Methods}

In addition to the investigation of repassivation on the nanoscale with an elapsed scratch depth versus time; conductance measurements were used as well. By combining the scratch methods developed in IV-AFM and the use of the AFM conductance module, further characterization and understanding of nanoscale repassivation of Ti-6Al-4V was possible. Through the characterization of the altered oxide over time directly represents repassivation at the implant oxide interface on the nanoscale. Over time as the oxide reforms the amount of repassivation can be observed and quantified both by surface characterization and difference in conductance measurements.

Therefore, the C-AFM experiments were developed to observe the ability of the AFM to capture repassivation on the nanoscale with conductance measurements. Utilizing conductive AFM techniques, C-AFM was broken up into three different versions A, B, \&C; (A) was 
performed in air at 20,37 , and $45^{\circ} \mathrm{C}$, (B) was performed in air at $20{ }^{\circ} \mathrm{C}$, and (C) was performed in-vitro with $\mathrm{HBBS}$ at $37^{\circ} \mathrm{C}$. With the following experiments, the ideal observation of repassivation was to monitor the current as a voltage bias was applied after scratches were performed, in hopes of witnessing at drop in conductance overtime as illustrated in Figure 2.8. This observation of a drop in conductance overtime would show the ability of the AFM to create and capture repassivation on the nanoscale with conductance measurements.

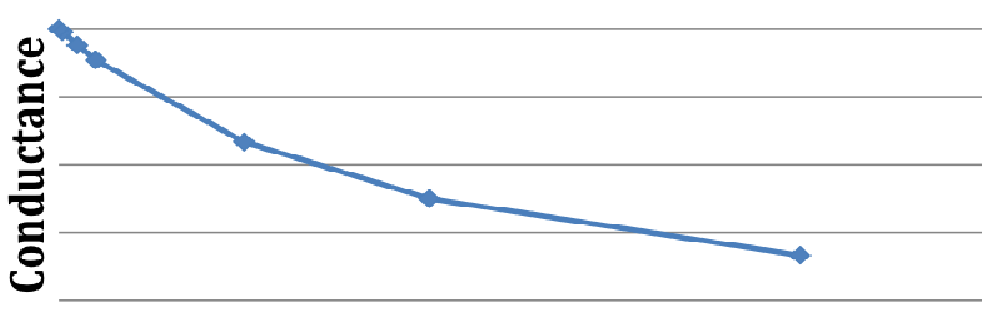

\section{Time (Min)}

Figure 2.8 Expected observations of conductance measurements after scratch is created with the AFM. The drop in conductance overtime represents the observation of repassivation around the created scratch.

Utilizing the same AFM scratch test methods used in IV-AFM experiments along with the implementation of conductive AFM techniques. The AFM allows for the integration of both zsensor data with conductance data. The software will be able to create images of surface topography as well as current distribution superimposed in the same image as demonstrated in Figure 2.9. 


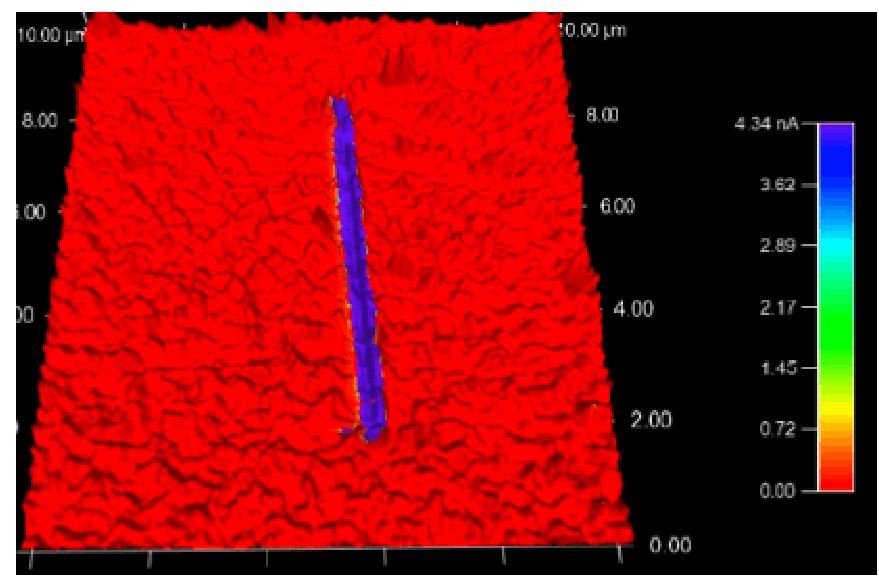

Figure 2.9 C-AFM AFM (10 $\mu$ m scan) current contrast image of current measurements overlaid on a $z$-sensor topography scan. Image clearly indicates current detection around scratch.

\subsubsection{C-AFM (A) Repassivation Test}

C-AFM (A) was designed to characterize repassivation with the use of conductance measurements. Similar to the IV-AFM experiments, all C-AFM experiments were performed in the materials engineering microscopy lab at California Polytechnic State University, San Luis Obispo. All experiments were performed using an Asylum Research MFP-3D AFM in conjunction with the BioHeater AFM accessory, MicroAngelo ${ }^{\mathrm{TM}}$ nanolithography software, Asylum Research ORCA ${ }^{\mathrm{TM}}$ conductive module, and diamond coated conductive cantilevers. The samples were also identically prepared and characterized with the AFM as in the previously performed IV-AFM experiments. Each experiment started with locating a testing area $(10 \mu \mathrm{m} x$ $10 \mu \mathrm{m})$ that was smooth, flat, debris free and allowed to equilibrate to each testing temperatures 20,37 , and $45^{\circ} \mathrm{C}$ prior to testing. To control the testing temperature of the sample the use of the Petri dish Bioheater was used. Temperature was also monitored and recorded with a thermocouple to verify actual testing temperature. For each temperature three replications were performed. In Figure 2.10 it can be seen that the actual testing temp was slightly lower than the targeted testing temp. This can be attributed to the BioHeaters reduction in performance when trying to heat air versus its indented use with fluids. 


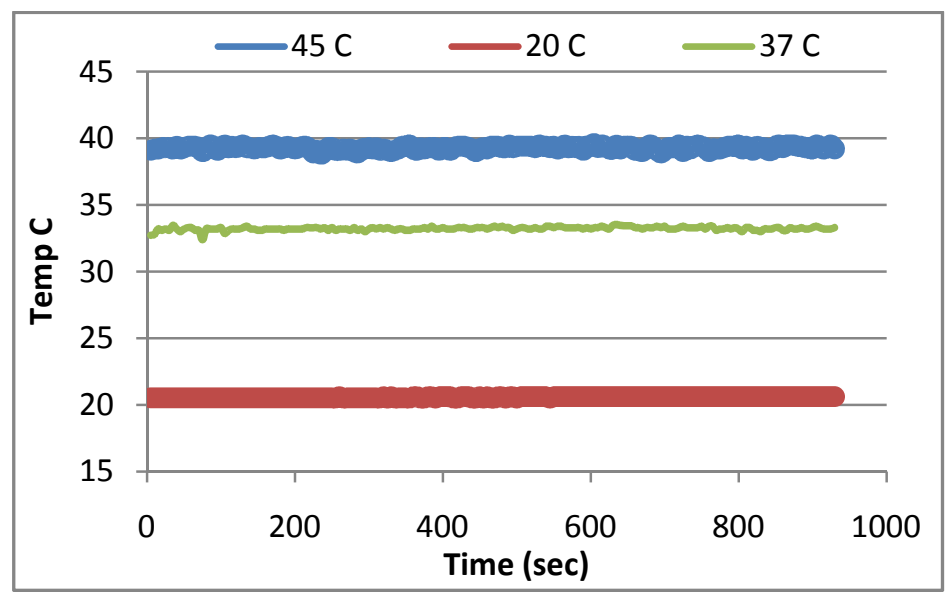

Figure 2.10 C-AFM (A) average temperature profile of experiments, showing lower testing temperatures than the desired target testing temperatures.

After locating a testing area and reaching equilibrium, the experiments started with an AFM surface characterization in AC mode prior to performing nano-scratch. Prior to testing a bias voltage of $3.63 \mathrm{~V}$ was applied until trace amounts of current could be detected before scratching through oxide. The characterization of the substrate as well as conductance measurements prior to scratching are regarded as the zero state of repassivation and provide the starting point to which each scan post scratching will be compared to. After characterization of the substrate the AFM lithography software was used to create a single $6 \mu \mathrm{m}$ scratch in the oxide substrate. The average applied force to create scratches was $\sim 30 \mu \mathrm{N}$ and varied depending on cantilever spring constant and surface morphology. For all experiments the NanoWorld ${ }^{\mathrm{TM}}$ CDT-NCHR diamond coated conductive cantilever was used with a $2 \mathrm{~Hz}$ scan rate. C-AFM (A) was designed to demonstrate the ability to perform nano-scratches and characterize there changes at different temperatures with the use of conductive AFM techniques in air. 


\subsubsection{C-AFM (B) Repassivation Test}

Using the same test methods as performed in C-AFM (A), conductance and z-sensor data was collected and replicated three times for each sample except for sample \# 7 where only two replications were performed due to a lack of cantilever supplies. The intent of C-AFM (B) was to characterize sample variation with the use of the collected conductance and z-sensor data. The conductance and z-sensor data was used to look for any discrepancies between the data sets to quantify variations within or between experiments determining repeatability in the experiments.

\subsubsection{C-AFM (C) In-vitro Repassivation Test}

In efforts of further understanding repassivation, the development of in-vitro C-AFM test methods were created. As performed in both (A) \& (B), (C) used the same over all AFM setup, but with all testing conditions simulated in-vitro, with the addition of HBSS. To create the invitro conditions, the BioHeater was used to maintain physiological temperatures were the addition of $10 \mu \mathrm{L}$ of HBSS was placed on the sample simulating physiological fluids. The AFM cantilever was then submerged in the created HBSS droplet, were the same nano-scratch test methods used in C-AFM (A) were performed.

\subsection{Cantilever Tip Characterization}

In order to investigate the performance of the cantilevers used, tip characterization was performed on one NanoWorld ${ }^{\mathrm{TM}}$ CDT-NCHR diamond coated conductive cantilever with the use of a Phillips FEI Quanta 200 scanning electron microscope (SEM). The cantilever was imaged under high vacuum with an electron beam voltage of $15-25 \mathrm{kV}, 3 \mu \mathrm{m}$ spot size, and a $45^{\circ}$ tilted sample stage. Several different magnifications were used for tip characterization. 


\section{RESULTS}

In this chapter the previously mentioned experimental procedures are presented and used to interpret the nano-scratch test methods performed on Ti-6Al-4V via AFM techniques. The following are objectives regarding the use of AFM and the observations that are thought to be associated with nano-repassivation.

1. Demonstrate the feasibility to create nano-repassivation conditions, as well as the ability to characterize in-vitro nano-repassivation via AFM techniques

2. Demonstrate the ability to characterize nano-repassivation in air, with the use of conductive AFM techniques

3. Demonstrate the ability to characterize in-vitro nano-repassivation with the use of conductive AFM techniques

\subsection{AFM In-vitro Repassivation Results}

\subsubsection{IV-AFM Experimental Results}

IV-AFM nano-scratch test methods were performed on anodized Ti-6Al-4V substrates creating simulated in-vivo repassivation conditions. Experimental scratches were performed in HBSS at $37{ }^{\circ} \mathrm{C}$ and observed with the AFM (z-sensor data) over time at 1 and $3 \mathrm{~Hz}$ scan rates (Figure 3.1 $a \& b$ ). With the use of AFM topography characterization (z-sensor data), the maximum in-vitro scratch depth was monitored for each set of experiments at both 1 and $3 \mathrm{~Hz}$ scan rates after scratches were created. The scratches in Figure 3.1 show the ability of the AFM to physically manipulate the surface penetrating the $(\sim 15-20 \mathrm{~nm})$ anodized oxide layer. The data suggests that the scratching ability is capable of reaching depths greater than the existing oxide layer. With this ability it can be assumed that the scratch depths were able to penetrate depths greater than the 
grown oxide, indicating that base material was exposed, creating corrosive conditions simulating in-vitro repassivation. Although, the AFM was capable of creating these scratches, the scratches did show some variation between experiments. In Figure 3.1 the scratches were initiated at the top of the image and ended at the bottom and were intended to be straight $6 \mu \mathrm{m}$ scratches.

However, as can be seen in the figure the scratches arc and show variable scratch depth profiles.
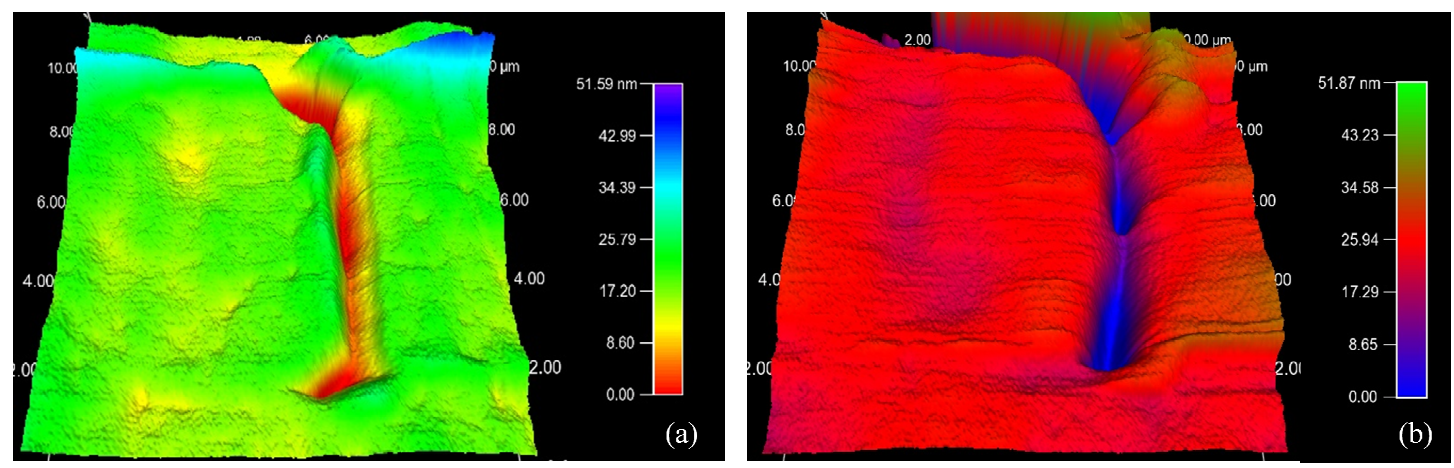

Figure 3.1 (a) AFM image of a nano scratch created in the $1 \mathrm{~Hz}$ IV-AFM (b) AFM image of a scratch created in the $3 \mathrm{~Hz} I V$-AFM experiment, where the color bar on the right shows a depth profile of approximately $30 \mathrm{~nm}$ for both experiments (Both images are $10 \mu \mathrm{m}$ scans).

This variability in scratching ability of the titanium oxide surface indicated that the variable could be the effect of the cantilever tip traversing through differing oxide compositional structures. To look for statistical variance within the experiments, one-way ANOVA was performed on the maximum scratch depths recorded for each scan during the experiments. With a confidence interval of $95 \%$ a p-value $<0.05$ was calculated, indicating that the results showed statistical differences in the created scratchs between experiments (Table V). 
Table V. One-way ANOVA on (1Hz) IV-AFM measured scratch depth ANOVA

\begin{tabular}{lcccccc}
\hline \multicolumn{1}{c}{ Source of Variation } & SS & $d f$ & $M S$ & $F$ & P-value & F crit \\
\hline Between Groups & 20927.5 & 2 & 10463.75 & 1776.512 & $1.45 \mathrm{E}-15$ & 3.885294 \\
Within Groups & 70.68065 & 12 & 5.890054 & & &
\end{tabular}

Total $\quad 20998.18 \quad 14$

The scratching ability was also observed with the measured scratch depths. The range of depths varied between experiments and varied from $25-120 \mathrm{~nm}$ in depth with an average depth of $\sim 73$ $\mathrm{nm}$. In each of the $1 \mathrm{~Hz}$ experiments, there was also little evidence of change in the monitored maximum scratch depth as shown in Figure 3.2. The data suggests that scratches were created to a variable depth, but no significant changes were observed in the maximum scratch depth overtime, indicating that the AFM was inadequate in observing repassivation by monitoring the scratch depth at the slow $1 \mathrm{~Hz}$ scan rate.

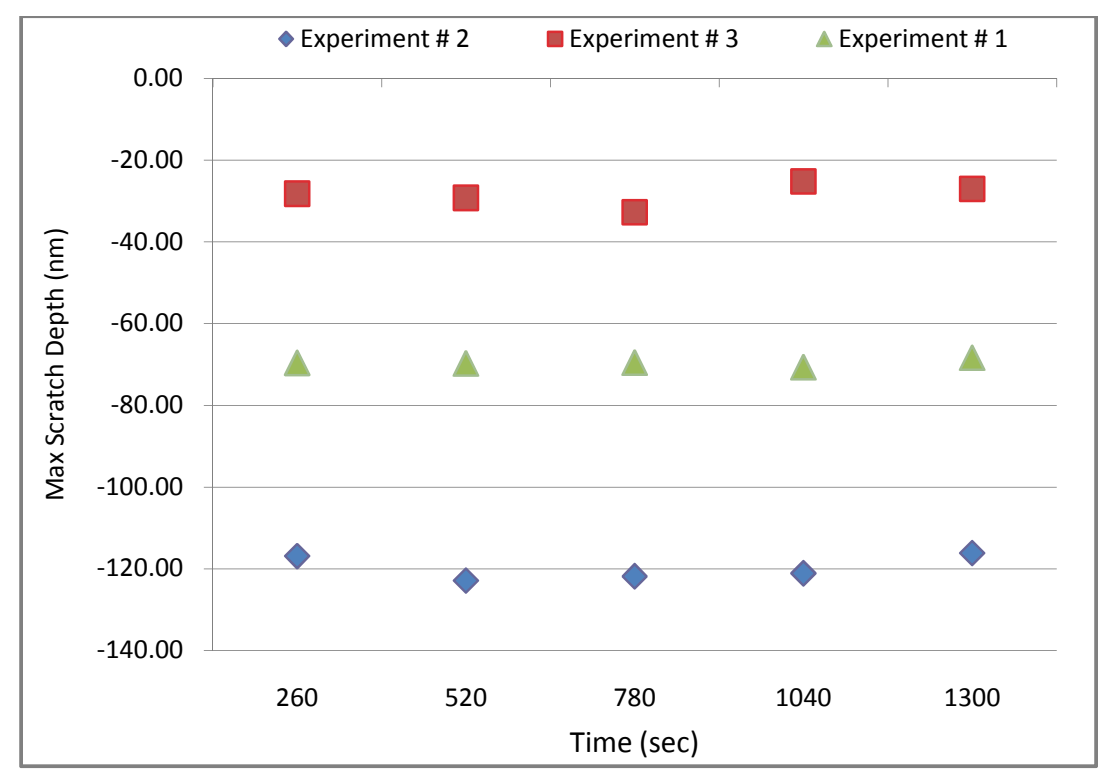

Figure 3.2 IV-AFM (1Hz) in-vitro max scratch depth that was monitored over time showing variance in scratch depth between experiments performed in $\mathrm{HBSS}$ at $37^{\circ} \mathrm{C}$. 
As performed in the $1 \mathrm{~Hz}$ IV-AFM experiments, $3 \mathrm{~Hz}$ data collection methods were performed after nano-scratches were created. There were more promising results with the faster scan rates. The scratch depths were more consistent, resulting in a smaller range of 52-68 nm maximum scratch depths in comparison to experiments performed at $1 \mathrm{~Hz}$. The data also suggests that the maximum scratch depth, were deeper than the anodized oxide layer $(15-20 \mathrm{~nm})$ indicating that anodized oxide layer was sufficiently removed in all experiments, exposing base material and creating repassivation conditions. Figure 3.3 also shows experiment \# 2 decreasing in maximum scratch depth over time. This indicates that the AFM was capable of observing minimal surface changes, after scratching. It is possible that these changes are representative of repassivation. Unfortunately, experiment \# 1 showed minimal if any change in the observed maximum scratch depth. The graph also only shows two replications as there were limited cantilever supplies to replicate the experiment a third time. Therefore, much like what was observed in the $1 \mathrm{~Hz}$ experiments, there is not enough evidence to justify the ability of the AFM to observe repassivation with topographical surface characterization (z-sensor data).

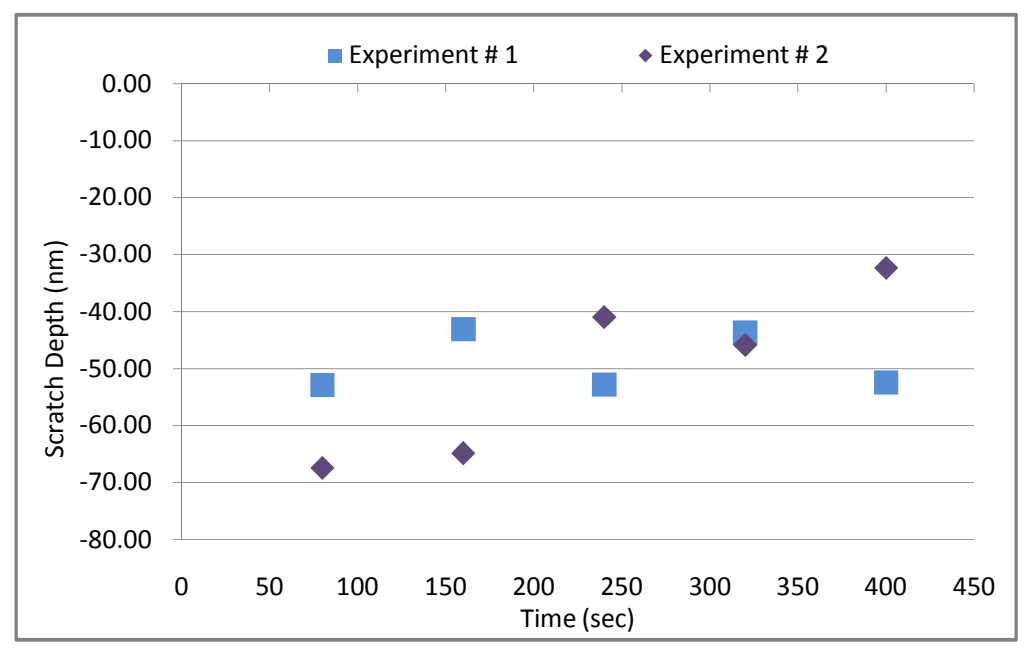

Figure $3.3 \mathrm{IV}-\mathrm{AFM}(3 \mathrm{~Hz})$ in-vitro scratch depth monitored over time graph showing small changes in scratch depth throughout the experiments performed in HBSS and controlled with the AFM at $37 \mathrm{C}$. 


\subsection{C-AFM Repassivation Results}

AFM scratches were performed on anodized Ti-6Al-4V substrates in air and in-vitro and were observed with the AFM z-sensor and conductance module (Figure 3.4). As with IV-AFM experiments, all C-AFM experiments were successful at creating scratches that were deep enough to penetrate the anodized oxide layer $(\sim 15-20 \mathrm{~nm})$ suggesting that repassivation conditions were met. However, since the same z-sensor characterization methods were proved to be inadequate in observing surfaces changes in IV-AFM, only observations of conductance will be presented for C-AFM experiments.

\subsubsection{C-AFM (A) Conductive Repassivation}

The AFM was successfully in taking conductive measurements with the conductive module. With the created nano-scratch test methods, the current measurement data showed evidence of altering the anodized oxide layer. Since the conductance measurements are taken simultaneously with the z-sensor data, the AFM software has the ability to superimpose current data on top of the topographical data showing exactly where the current was measured (Figure 3.4). As performed in Figure 3.4 all scratches were created with the cantilever perpendicular to the created scratch. With this orientation of the cantilever during scratching it is believed that the tip in this orientation is the source of the scratches tailing off at each end. Regardless of the tailing, the created scratches show evidence that the AFM tip was successful in penetrating the oxide layer allowing current to flow in and around the created scratches. All current data was taken with an applied $3.63 \mathrm{~V}$ bias, as this was the necessary breakdown potential of the oxide to allow electron transport through the oxide before manipulation of the oxide. 

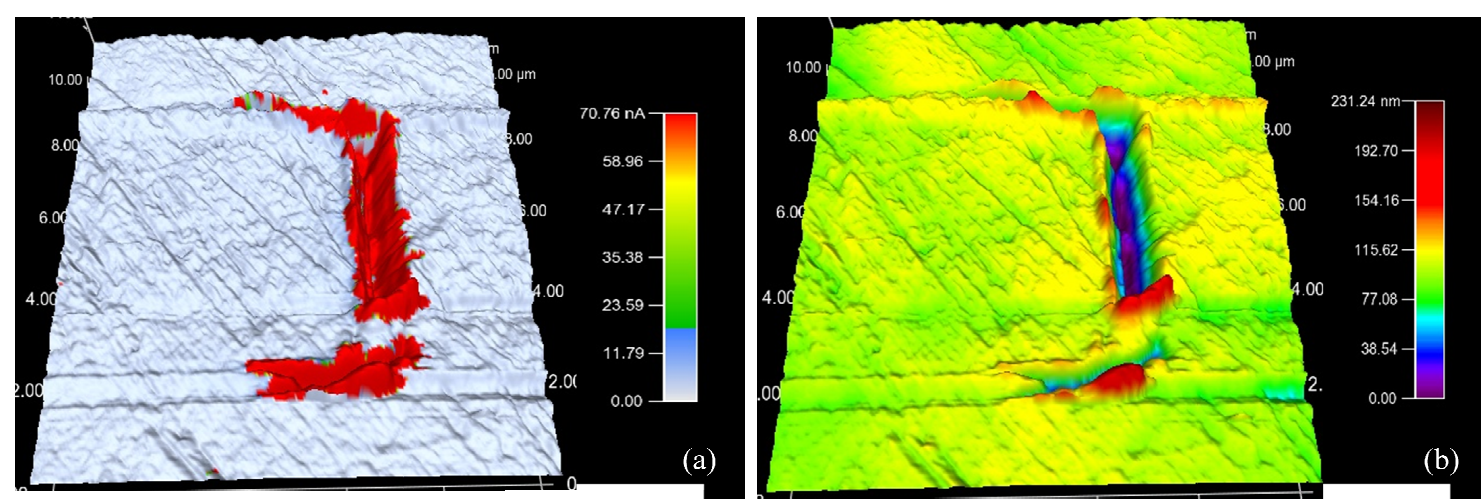

Figure 3.4 (a)C-AFM (A) z-sensor profile image with current measurements superimposed on scanned topography. (b) Same image as (a), but only showing z-sensor depth of scratch with color bar showing scratch depth (10 $\mu m$ AFM scan).

\subsubsection{C-AFM (A) Conductive Repassivation Results at $20^{\circ} \mathrm{C}$}

Conductance measurements were observed after scratching with an applied $3.63 \mathrm{~V}$ bias at 20

${ }^{\circ} \mathrm{C}$ (Figure 3.5). From the data it can be seen that average current measurements were observed within a range of $2-4.5 \mathrm{nA}$. This clearly shows the capability of the AFM to successfully observe current measurements within the scratch after nano-scratch test methods were performed.

Looking at the data closer, a trend in the reduction of current over time with the applied $3.63 \mathrm{~V}$ bias was also observed and calculated to be $-.006 \mathrm{nA} / \mathrm{sec}$. This reduction in current over time seems to follows the expected trend as described in chapter 2, but is not direct evidence of repassivation. The amount of variance was also plotted and shows that over time the amount of variance in current measurement decreased over time (Figure 3.5b). 

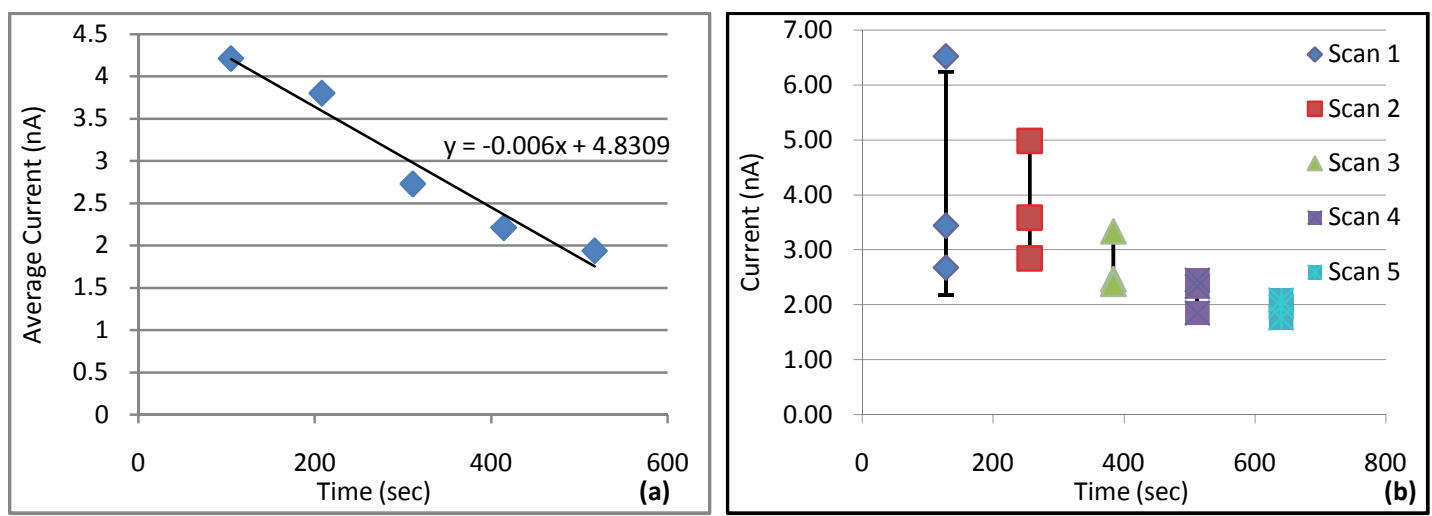

Figure 3.5 C-AFM (A) experiments performed with 3.63V bias at $20 \mathrm{C}$. (a) Is the average current measurements over time after AFM nano-scratches were created on Ti-6Al-4Vsubstrates and (b) is the amount of variance between replicated experiments. Measured slope is rate of decreasing current and error bar are standard deviation.

\subsubsection{C-AFM (A) Conductive Repassivation Results at $37^{\circ} \mathrm{C}$}

At $37^{\circ} \mathrm{C}$, conductance measurements were observed with an applied $3.63 \mathrm{~V}$ bias as the data was collected at $2 \mathrm{~Hz}$ scan rate. As performed at $20^{\circ} \mathrm{C}$, the data indicates the capability of the AFM to successfully measure observable changes in current measurements after performing nano-scratch test method. Figure 3.6 shows the plotted data over time where it shows the average current measurements in a range of $\sim 18.8-19.1 \mathrm{nA}$ and a $-.002 \mathrm{nA} / \mathrm{sec}$ decreasing rate of current. It was also noticed that with the increased testing temperature, the current measurements increased as well. This indicated that temperature had an effect on the observable current measurements. Although, with the observed increase in current detection the rate at which the current decreased was comparable to what was observed at $20^{\circ} \mathrm{C}$. Variance was plotted and shows the greatest amount of variance in the first scan and then decreases over time (Figure 3.6b). However, these observations of decreasing rates of current and variance over time, these experiments cannot be directly associated with repassivation and will be further discussed in detail in chapter 4 . 

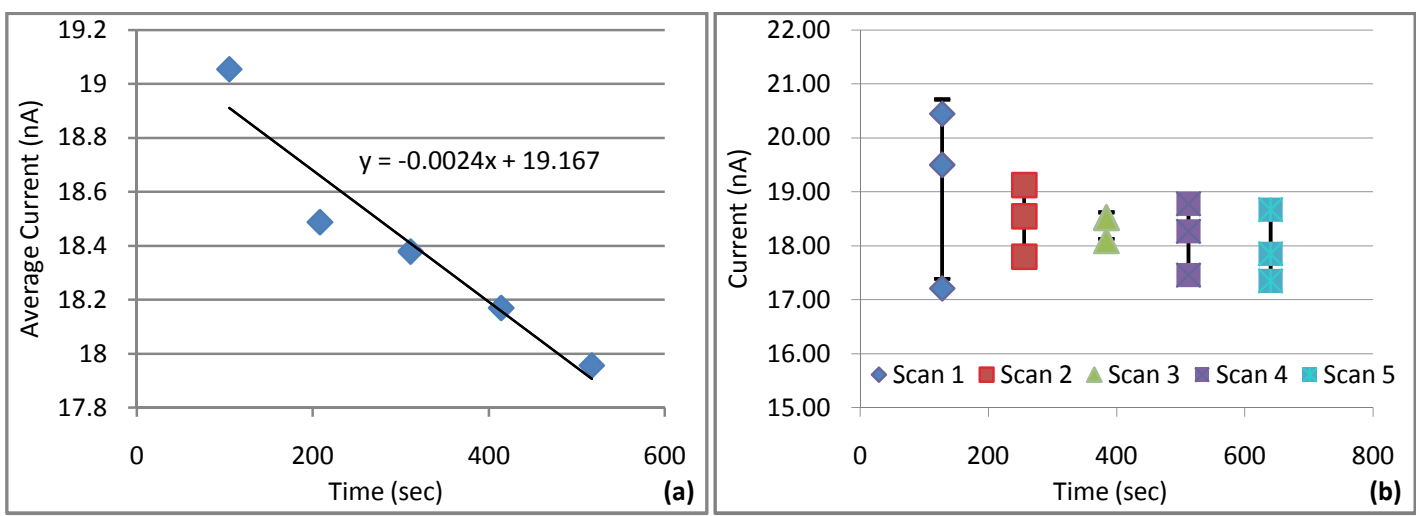

Figure 3.6 C-AFM (A) experiments performed with 3.63V bias at $37 \mathrm{C}$. (a) Is the average current measurements over time after AFM nano-scratches were created on Ti-6Al-4Vsubstrates and (b) is the amount of variance between replicated experiments. The measured slope is the rate of decreasing current and error bar are standard deviation.

\subsubsection{C-AFM (A) Conductive Repassivation Results at $45^{\circ} \mathrm{C}$}

As were performed at 20 and $37^{\circ} \mathrm{C}$, there were no differences in the experimental methods other than the associated $45^{\circ} \mathrm{C}$ testing temperature. As can be seen in Figure 3.7, the average current measurements were measured within a range of 15-16.8 nA. Again, with an increase in temperature, an increase in current measurement was observed. Although, at a higher temperature than the previously performed $37^{\circ} \mathrm{C}$, the average measured current was lower, but the rate of decreasing current was $-.004 \mathrm{nA} / \mathrm{sec}$ indicating that it was higher. However, if you look the variance associated between the experiments (Figure 3.7b), it becomes evident that one of the three replicated experiments had experimental error. Therefore, with the presence of excessive variance the calculated average current measurements were affected. In the experiments it was observed that one of the tests performed had substantially lower current measurements than the two previously performed tests. This lower measurement is believed to be caused by replicating the scratch experiments with the same cantilever. This may have caused enough damage to the cantilever tip between experiments to compromise conductivity measurements. Tip characterization was performed on one of these tips pre and post scratching and will be further discussed in chapter 4 . 

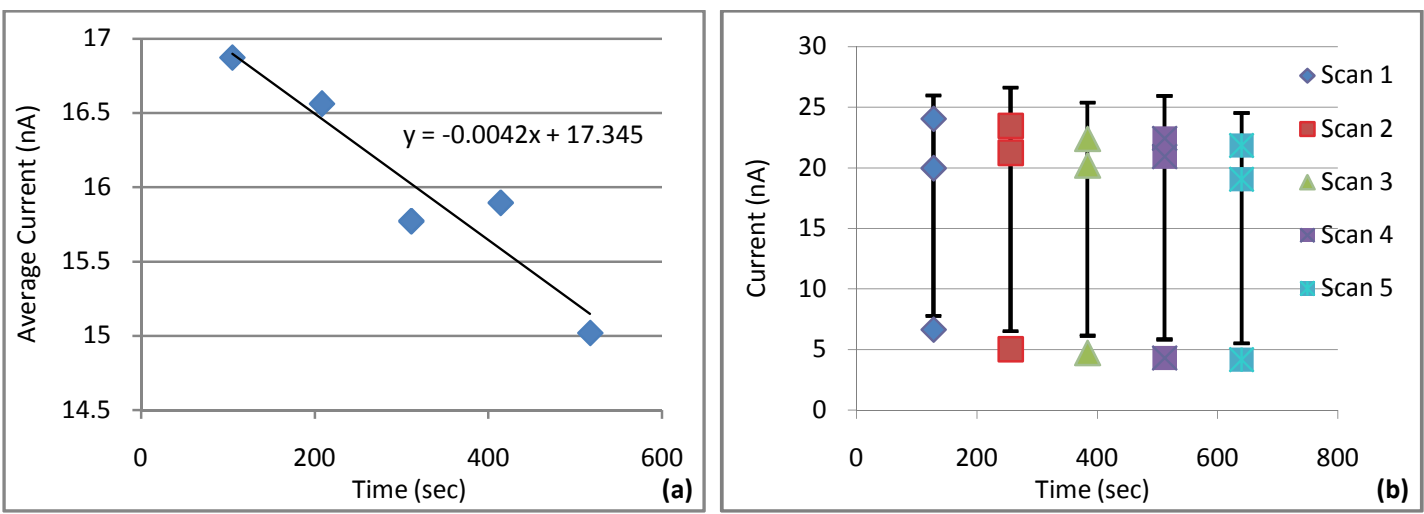

Figure $3.7 C$-AFM (A) experiments performed with $3.63 \mathrm{~V}$ bias at $45 \mathrm{C}$. (a) Is the average current measurements over time after AFM nano-scratches were created on Ti-6Al-4Vsubstrates and (b) is the amount of variance between replicated experiments. Measured slope is rate of decreasing current and error bar are standard deviation.

\subsubsection{Temperature Effects on C-AFM (A) Results}

As mentioned earlier in the C-AFM (A) results, it was observed that at the three different experimental temperatures, the current measurements increased. From Figure 3.8, it shows the differences in current measurements with each associated temperature.

With the increased temperature the average current measurements were higher than the previously performed experiments at lower temperatures. This indicated that with the added thermal energy, the current was more capable of flowing through the substrate (oxide) of the sample increasing its conductivity. Further discussion about the effect of temperature and conductivity will be discussed in chapter 4 . 


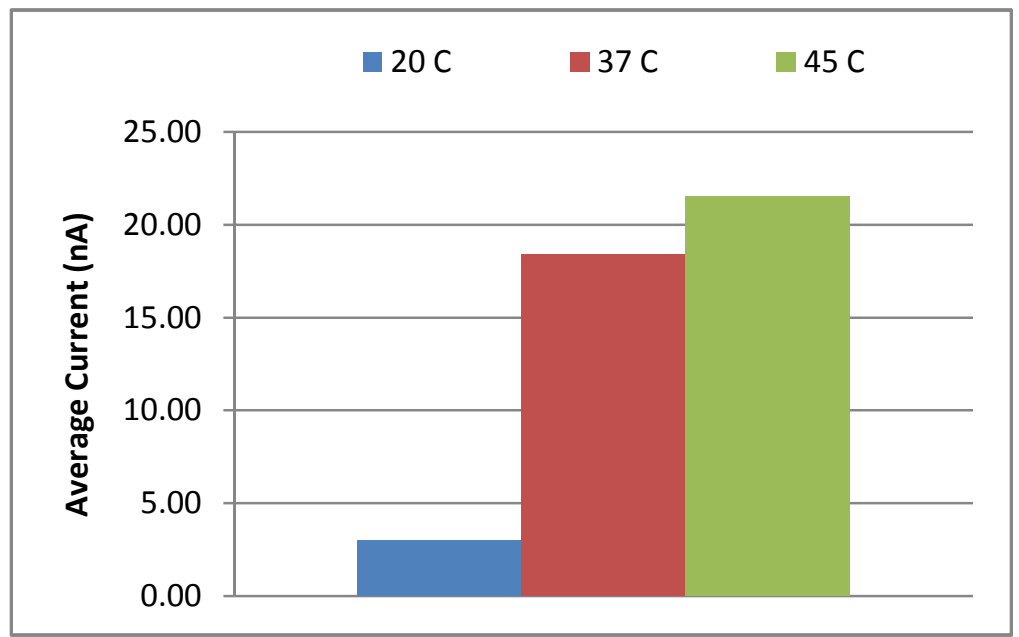

Figure 3. 8 C-AFM (A) bar chart showing an increase in overall current detection with an increase in testing temperature.

\subsubsection{C-AFM (B) Conductive Repassivation Results}

C-AFM (B) was able to take conductive measurements after performing nano-scratch test methods on Ti-6Al-4V samples. The only difference in comparison to C-AFM (A) was the fact that all C-AFM (B) experiments were replicated over three different samples at $20{ }^{\circ} \mathrm{C}$. By replicating the experiment the intent was to create a larger population to look for variation in the experiments as well as in the samples. A total of eight experiments were performed over three different samples. Figure 3.9, shows the range of current measurements between the first and last experiments, where all other experiments fell within these two experiments. The graph also shows that all experiments were measured under $0.7 \mathrm{nA}$. 


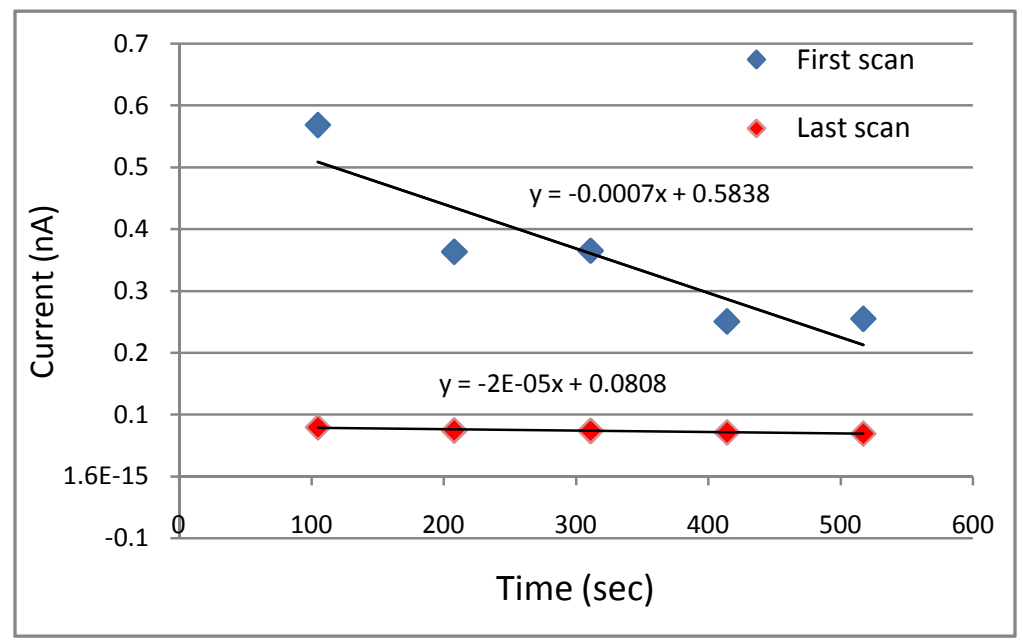

Figure 3.9 Range of average current measured in C-AFM (B) experiments over time after AFM manipulation of Ti-6Al-4Vsubstrates with a 3.63V bias applied at $20 \mathrm{C}$.

Recalling what was observed in C-AFM (A) at $20^{\circ} \mathrm{C}$, the values in Figure 3.9 are approximately a tenth of what was measured in that experiment. Along with decreased current measurements, another observation was noticed that the overall current measurements showed a progressive decline in the observable current detection. The rate of decreasing current also decreases from $.0007-.0002 \mathrm{nA} / \mathrm{sec}$ indicating a loss in conductance performance. In Figure 3.9 although, the current values are low, the initial experiment shows the desired trend in a reduction in current over time. Where as in the last C-AFM (A) experiment, the values are minimally detected and show no change in current measurements as if the conductivity of the cantilever tip was being degraded with each experiment. Reasons for these values are believed to be attributed to cantilever quality such as having initial tip damage prior to testing. It should be noted that the cantilever used for these experiments was characterized before and after all completed tests.

Analyzing the data, one-way ANOVA was performed on the average current measurements with a $95 \%$ confidence interval and resulted in a p-value $<0.05$ (Table VI). In the analysis a large F- value was calculated indicating that the variation among groups is more than you'd expect to see randomly. By also calculating $\mathrm{R}^{2}$ percentages, $\sim 84 \%$ of the variance can also be associated 
to be between group variations. This suggests that sample to sample variance was a major factor in the outcome of the experiment. These variances within the groups are most likely derived from the initial variance introduced in the sample prep or may be caused by the diminishing performance of the cantilever tip. Further discussion will be addressed in chapter 4 .

Table VI. One-way ANOVA on C-AFM (B) average measured current

\begin{tabular}{|c|c|c|c|c|c|c|}
\hline Source of Variation & $S S$ & $d f$ & $M S$ & $F$ & P-value & $F$ crit \\
\hline Between Groups & 0.90932 & 7 & 0.1299029 & 23.54486435 & $6.28 \mathrm{E}-11$ & 2.3127412 \\
\hline Within Groups & 0.1765519 & 32 & 0.0055172 & & & \\
\hline Total & 1.0858719 & 39 & & & & \\
\hline
\end{tabular}

\subsubsection{C-AFM (C) In-vitro Repassivation Results}

With numerous attempts using the nano-scratch test method in parallel with the AFM conductance module, C-AFM (C) was unsuccessful in observing changes post scratching. The use of HBSS reduced the ability to detect repassivation current. The ions present in the HBSS caused current measurements to be greater than the $100 \mathrm{nA}$ meter capacity of the conductance module. In an effort to reduce the amount of current measured, added resistance to the conductive circuit was performed. However, the addition of the resistance was not enough to establish an offset of the actual current detection. During engagement of the cantilever into the fluid and on to the substrate the current detection meter maxed out without a voltage bias being applied. Therefore, with the current meter in the conductance module being maxed out, resolvable measurements of current were not capable of being performed. In comparison to the previous C-AFM experiments, Figure 3.10 shows what the current measurement looked like in relationship. From the images it is clear that current measurements were off the chart throughout the entire sample in C-AFM (C) experiment (Figure $3.10 \mathrm{a}$ ). Figure $3.10 \mathrm{~b}$, shows the current isolated around the created scratch. 

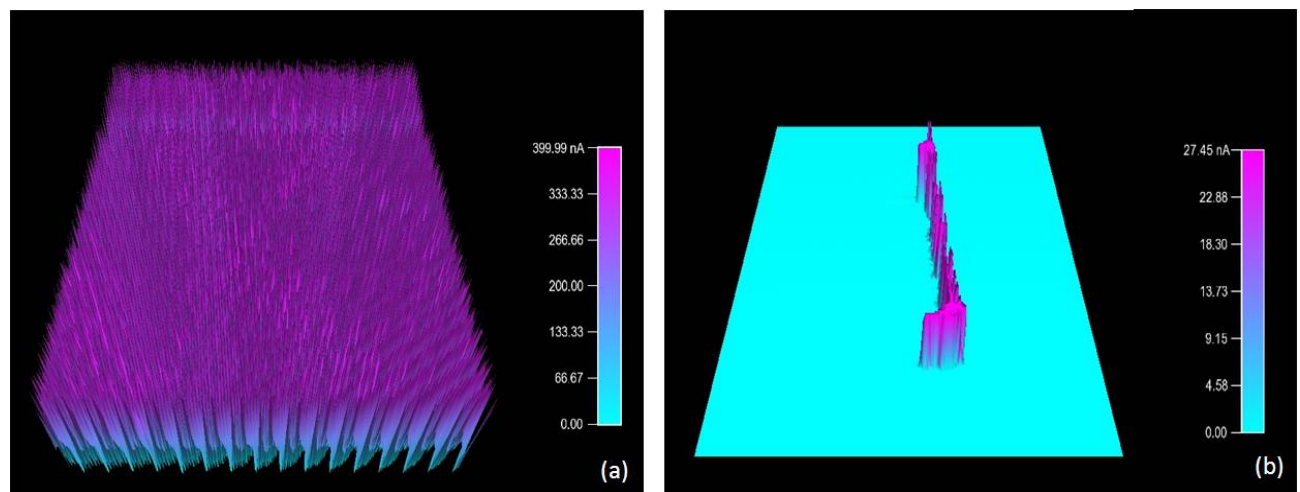

Figure 3. 10 (a) AFM current measurements in C-AFM C in HBSS at 37 C and (b) Current measurements in C.AFM (A) in air at 37 C. (Roth imoges are 10 um AFM scans)

\subsection{SEM Cantilever Tip Characterization}

Using the SEM, cantilever tips were characterized, pre and post experiments. Characterization included measurements of the radius of curvature which was found to be $\sim 170 \mathrm{~nm}$, comparable to the published value range of 100-200 $\mathrm{nm}$. The post-experiment radius of curvature was measured to be $\sim 900 \mathrm{~nm}$ and showed evidence of wear, blunting of the tip, along with debris around the tip, as if remains of the scratched substrate were smeared on the end of the cantilever tip (Figure 3.11). These images show the effects of the lithography performed throughout these experiments and suggest that cantilevers be exchanged between samples or if possible between experiments.

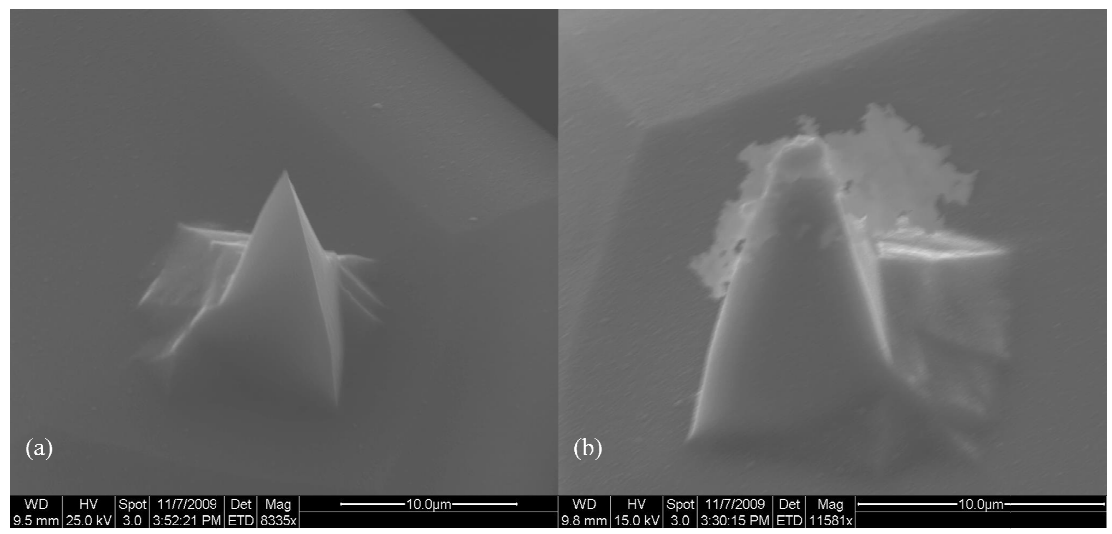

Figure 3. 11 AFM cantilever images comparing pre and post experiments. (a) is a image of an unused cantilever at $8335 x$. (b) is the same cantilevers post experiments at 11,581 $x$ showing evidence of tip damage during experiments as well as debris left on the tip. 


\section{DISCUSSION}

\subsection{Overview of Objectives}

In the results section, several experiments were attempted to meet the research objectives regarding the characterization of nano repassivation on Ti-6Al-4V via AFM nano-scratch test techniques. The following objectives investigated the ability of the AFM to simulate in-vivo orthopaedic repassivation.

1. Demonstrate the feasibility to create nano-repassivation conditions, as well as the ability to characterize in-vitro nano-repassivation via AFM techniques

2. Demonstrate the ability to characterize nano-repassivation in air, with the use of conductive AFM techniques

3. Demonstrate the ability to characterize in-vitro nano-repassivation with the use of conductive AFM techniques

\subsection{Evaluation of In-vitro Repassivation via AFM Z-Sensor Data}

In-vitro nano-scratch test methods were conducted to create and characterize the repassivation kinetics of anodized Ti-6Al-4V.

\subsubsection{In-vitro Repassivation (IV-AFM)}

The development of AFM in-vitro experiments were attempted as innovative techniques to characterize nano-repassivation on Ti-6Al-4V substrates with AFM z-sensor scans. In-vitro repassivation experiments are not new by any means. Previous research has been performed to characterize repassivation of implants. Repassivation scratch tests at the macro scale have been used to simulate in-vitro oxide removal. These methods observe repassivation on a larger scale, 
rather than the nanoscale where repassivation is theorized to occur (Milosev, Hukovic, \& Strehblow, 2000).

With the results presented in the IV-AFM experiments, it was shown that the AFM was capable of an average scratch depth of $\sim 73 \mathrm{~nm}$, which was more than adequate to penetrate the $\sim 15-20$ nm oxide layer exposing the base material. Although the experiments were able to create repassivation conditions, the ability to observe repassivation was limited. Due to the limitations of the scanning speed of the AFM scanner, results indicated that the AFM was incapable of observing changes in the surface topography with z-sensor data sufficiently fast in HBSS. It became apparent that the AFM was too slow to observe the reactions of repassivation. Since repassivation is time dependent the scratching time delays (48seconds) and the scanning speed (80-280 sec/scan) were the main reasons why the AFM was unable to capture the spontaneous reaction of repassivation. Therefore, it is suggested that future experiments utilize the scratching ability of the AFM to create repassivation condition, but characterize repassivation through some other means like using open circuit potentials (OCP) in conjunction with the AFM nano-scratch methods. With OCP experiments, the open circuit is the potential of the working electrode relative to a reference electrode. So, when a potential is applied relative to the open circuit, the system measures the OCP before a voltage potential is applied to the cell. Then applying a potential relative to that measurement, changes in current measurements can be compared and observed.

It was also mentioned that IV-AFM experiments had issues of variance in the results of the experiments. The cause of this variance is most likely due to the sample prep methods. With the measured surface roughness post polishing and anodizing, the data suggested that the samples were statistically different, indicating that the hand polishing and single sample anodization methods introduced oxide variation. Although, this variability was minute, due to the experiments being performed on the nanoscale, it showed that small variations can have greater effects on the nanoscale. In addition to the polishing methods, the use of the individual anodization process was 
also a contributing factor to the associated variance. A batch process where all samples are submerged simultaneously may have been a more controllable method that would reduce the amount of sample-to-sample variability providing more consistent oxides.

Cantilever selection was also a contributing factor to variance. After using the PPP-NCVH and having difficulty tuning and imaging with the cantilevers in HBSS, it was discovered that there might have been more optimal cantilevers available that have greater strength and durability to endure the nano-scratch test methods. Veeco Inc. makes a high spring constant three sided pyramidal single crystalline diamond tip mounted on a stainless steel cantilever, which has been proven to be successful in performing scratch tests on silicon substrates however; it is not conductive (Santinacci, Zhang, \& Schmuki, 2005).

\subsection{Evaluation of Repassivation via Conductive AFM Methods}

\subsubsection{C-AFM (A)}

The overall trend observed in the conductance measurements performed in C-AFM (A) experiments, was a decreasing amount of current over time. These results were what were to be expected with the reformation of the oxide layer acting as a kinetic barrier to the electrochemical process of repassivation. To verify these observations, the current measurements were used to back calculate current density, conductivity, and electron mobility. Current density was calculated with

$$
j=\mathrm{I} / \mathrm{A}
$$

Where (I) was the measured current and (A) was the estimated scratch area that current could flow through. For these calculations it was assumed that the scratch area was $\sim 2.4 \mathrm{E}-8 \mathrm{~cm}^{2}$. This area was estimated using the $6 \mu \mathrm{m}$ scratch length and approximated scratch width of $400 \mathrm{~nm}$ after scratching. After calculating the current densities for each of the C-AFM (A) experiments (Figure 4.1), the graph shows a decrease in current density over time, which is to be expected, as the 
current density is directly proportional to the amount of current measured. Comparatively, the measured values of $0.9-0.1 \mathrm{~A} / \mathrm{cm}^{2}$ do not correlate to $1.0 \mathrm{E}-04-1.0 \mathrm{E}-07\left(\mathrm{~A} / \mathrm{cm}^{2}\right)$ current densities observed on titanium using OCP (Contu, Elsener, \& Bohni, 2004).

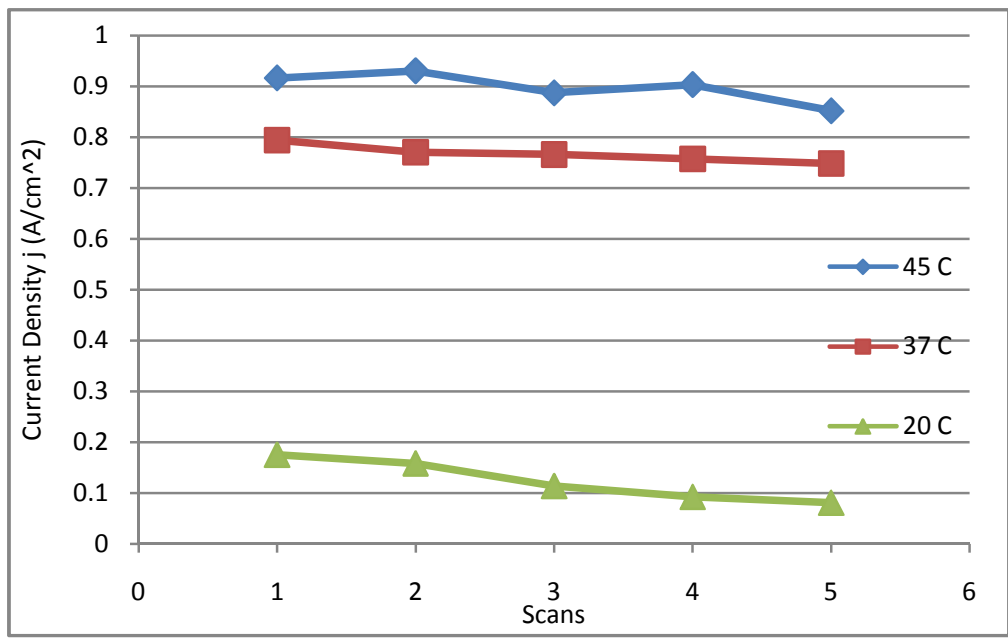

Figure 4. 1 Graph of estimated current densities observed in C-AFM (A) experiments

However, using these calculated values of current density, conductivity was calculated. Conductivity $(\sigma)$ is a function of electric field strength $(\xi)$ and current density $(j)$.

$$
\sigma=\xi / j
$$

Using the applied $3.63 \mathrm{~V}$ bias for $(\mathrm{V})$ and using the length $125 \mu \mathrm{m}$ of the cantilever as the conductive length (L), the electric field strength $(\xi)$ was determined to be $\sim 290 \mathrm{~V} / \mathrm{cm}$.

$$
\xi=\mathrm{V} / \mathrm{L}
$$

The conductivity was then calculated and plotted over time (Figure 4.2). From the graph it shows a decreasing conductivity for each experiment, which leads you to believe that repassivation is occurring, recreating the oxide and acting as a kinetic barrier to the flow of electrons. 


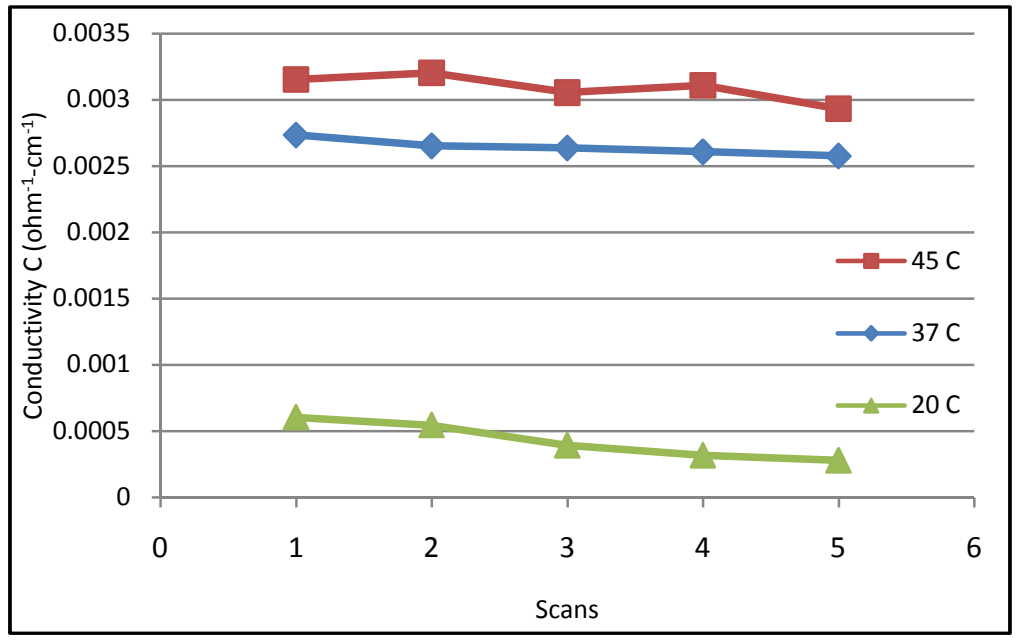

Figure 4. 2 Conductivity plotted over time showing a decreasing conductance and representing the observation of repassivation in $C-A F M(A)$ performed in air at corresponding temperatures.

Knowing this the calculated conductivity was compared to other common materials and was found to be more representative of a semi-conductive material. In the case of Ti-6Al-4V metallic oxides are generally considered insulative materials and, are primarily composed of $\mathrm{TiO}_{2}($ Ask, Lausmaa, \& Kasemo, 1989). Conversely, $\mathrm{TiO}_{2}$ has been studied to have semi-conductive properties and at room temperature was approximately measured to be $.003-0005 \Omega^{-1}-\mathrm{cm}^{-1}$ (Mardare, Baban, Gavrila, Modreanu, \& Rusu, 2002).

Electron mobility $\left(\mu_{\mathrm{e}}\right)$ is directly correlated to $\sigma$ with the following equation

$$
\mu_{\mathrm{e}}=[\sigma] /[n|e|]
$$

and was calculated to support the observations of repassivation. Where $n$ is the number of free electrons $\left(\sim 10^{22}-10^{23} / \mathrm{cm}^{2}\right)$ and $e$ is the electrical charge of an electron $\left(1.6 \times 10^{-19} \mathrm{C}\right)$. Figure 4.3 agrees with the calculations of conductivity and shows electron mobility decreasing. 


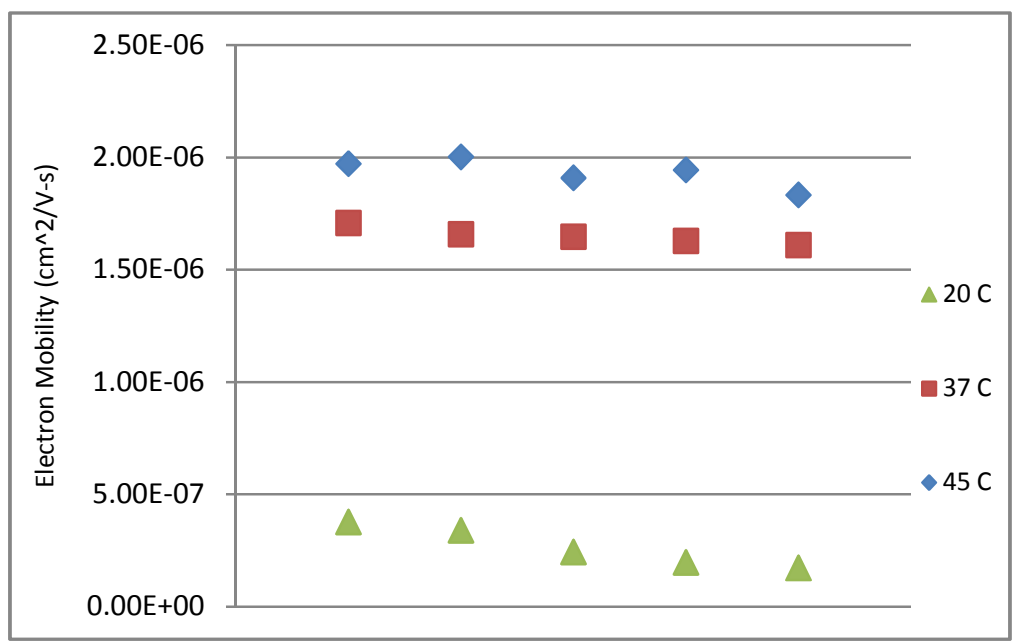

Figure 4. 3 Graph of electron mobility, using the current data in C-AFM (A) experiments showing a reduction in electron mobility as repassivation occurs.

As a result, these observations of the current detection present evidence of the ability of the AFM to capture current measurements associated with nano-scratch test methods. However, based on the data, it does not show a relationship to repassivation. Rather it is more representative of oxide growth and not the electrochemical reactions of repassivation. The measured electron mobility in Figure 4.3 are nearly identical to what has been observed in other studies specifically looking at the conductivity and electron mobility of thin film titanium oxides (Mardare, Baban, Gavrila, Modreanu, \& Rusu, 2002), (Aduda, Ravirajan, Choy, \& Nelson, 2004).

In conclusion, to observe and quantify repassivation through the means of measured current density, conductivity, and measured electron mobility the measurements observed should be more drastic and observed over a shorter time scale as were observed in other repassivation studies (Contu, Elsener, \& Bohni, 2004), (Goldberg \& Gilbert, 1998) (Gilbert, Buckely, \& Lautenschlager, 1996). However, C-AFM (A) did show the ability to successfully measure the conductivity and electron mobility of $\mathrm{TiO}_{2}$ and further supports the studies of Mardare et al. and Aduda et al. Studies that indicate $\mathrm{TiO}_{2}$ are better represented as a semi-conductive material and that further studies of utilizing their semi-conductive properties should be considered. 


\subsubsection{C-AFM (B)}

The magnitude of the current measurements in C-AFM (B) is questionable. With all current measurements under $.7 \mathrm{nA}$, it is speculated that some form of error was introduced into the experiments. The cantilever used for all C-AFM (B) experiments was characterized in the SEM. From the characterization the cantilever looked to be in perfect working order, and had a radius of curvature that was measured to be $170 \mathrm{~nm}$ which was within $10 \%$ of the manufactures published value $200 \mathrm{~nm}$. However, the current measurement results indicate otherwise with low current measurements under .7 nA and an overall observed current measurement decay. Therefore, there is strong belief that before the experiments were performed, the cantilever may have been damaged during handling to the extent that it altered the current detection capabilities. It is also possible that the scratch performed in the first experiment, may have damaged the tip to the extent of effecting conductance. Unfortunately, these theories cannot be explained with the data collected and can only be theorized as the tips were not analyzed before every experiment.

However, a single cantilever was characterized in the C-AFM (B) experiments prior to use and following the eight experiments it was used in. The characterized cantilever showed substantial wear and damage to the tip. The reason for the excessive use of a single cantilever was due to limited cantilever supplies. With the replicated use of the same cantilever in all 8 experiments, lead to excessive tip wear as indicated by the SEM characterization, and low current measurements throughout the conducted experiments. Therefore, with the tip characterization showing that physical tip abuse was evident during scratching it is likely the reason behind the reduced current measurements.

\subsubsection{C-AFM (C) In-vitro Repassivation}

Observations of in-vitro repassivation have been performed with current transient measurements, OCP's, and polarization tests to capture observations of repassivation on the macro scale (Gilbert, Buckely, \& Lautenschlager, 1996), (Contu, Elsener, \& Bohni, 2004). 
However, none of these experiments have been attempted with the use of conductive AFM techniques or attempted at the nano-scale. Unfortunately, the results of the C-AFM (C) experiments were not as successful as anticipated and require further refinement. The lack of ability to take measurements in a conductive fluid was the main reason why C-AFM (C) was unsuccessful. Futile attempts to add resistance in series to the testing circuit was attempted. In efforts of filtering the excessive current measurement before it was measured in the conductance module. Initially it was believed that the surrounding BioHeater or the AFM scanner itself may have created stray currents in the HBSS maxing out the $100 \mathrm{nA}$ meter in the conductance module. However, upon further investigation, it is believed that the reasons for the excessive amounts of current in the HBSS, maybe due to the excessive ions present increasing the mobile charge carriers available in the solution. The excess of mobile carriers act as a current amplifier, causing the amount of current flow to over load AFM conductance module. The cause is still uncertain however, if these current measurements can be isolated and filtered; future experiments can utilize conductive AFM to characterize nano-repassivation. Nevertheless, much has been gained in the attempts to characterize nano-repassivation with the use of conductive AFM.

\subsubsection{C-AFM (A) vs. (B)}

Although, the methods used for both C-AFM experiments were the same, there were significant differences between the results. Looking at the difference in current detection magnitude, C-AFM (A) was approximately 10 orders of magnitude higher than the data collected in the C-AFM (B) experiments. It was also noted that in C-AFM (A), new cantilevers were used with each temperature in comparison to C-AFM (B) where the same cantilever was used for all experiments due to limited quantities of cantilevers. As mentioned previously, it was apparent that in C-AFM (B), there was convincing evidence that the AFM tip may have been compromised before testing was performed. Therefore, with evidence of tip wear associated with these experiments, it 
becomes apparent that due to the probable miss fortune of the cantilever used in C-AFM (B), the two experiments cannot be compared. It is then suggested that further experiments be performed to quantify the current measurements associated with these AFM repassivation test methods and that new cantilevers be used with each experiment and characterized pre and post to quantify and observe tip effects.

\subsubsection{Temperature Effects on C-AFM}

With the C-AFM (A) experiments being performed at 20,37 , and $45^{\circ} \mathrm{C}$ it was observed that as experiments were performed at increasing temperatures, the average measured current increased. It is known that with increased temperature, thermal energy is added to a system through increased atomic vibration. This available thermal energy causes atoms to vibrate in metals and scatters electrons causing them to lose their kinetic energy and causing them to change their direction (Callister, 2003). This scattering effect can be quantified with electron mobility and correlates to the frequency of scattering electrons. Therefore, as temperature increases it should be observed that electron mobility would decrease as the added thermal energy would increase the frequency of scattering electrons.

However, with the observed increase in current measurements with the increase in temperature, the values of electron mobility and conductivity increase as well. In general, this should not be true for metals as with increased temperature conductivity decreases as the amount of electron carriers are decreased. However, these occurrences are commonly associated with semiconductive materials, where the effects of temperature cause the material to increase the amount of charge carriers' available allowing electrons to more readily flow (Askeland \& Phule, 2006). Given that the data indicated that electron mobility and conductivity increased with an increase in temperature. It is evident that the measurements are of a semi-conductive material and are representative of the electrical properties associated with $\mathrm{TiO}_{2}$ (Aduda, Ravirajan, Choy, \& 
Nelson, 2004). Therefore, to conclude if the data was in fact representative of $\mathrm{TiO}_{2}$ Equation 4.4 can be used to plot electrical conductivity of an intrinsic semiconductor versus temperature.

$$
\sigma=n_{o} q\left(\mu_{\mathrm{e}}+\mu_{\mathrm{h}}\right) \exp \left(-\mathrm{E}_{\mathrm{g}} / 2 k \mathrm{~T}\right)
$$

Using the data from the conductivity measurements the data was plotted and fit with a logarithmic equation (Figure 4.4). The equation was then used to determine an experimental parameter $\sigma_{\mathrm{o}}$, and was set equal to $n_{o} q\left(\mu_{\mathrm{e}}+\mu_{\mathrm{h}}\right)$ leaving the expression .

$$
\sigma=\sigma_{\mathrm{o}} \exp \left(-\mathrm{E}_{\mathrm{g}} / 2 k \mathrm{~T}\right)
$$

From the equation in Figure $4.4 \mathrm{~b}$ the parameter $\sigma_{\mathrm{o}}$ was calculated to be .0025 and using Boltzmann's constant $(k)$ and the testing temperature $(\mathrm{T})$ a band gap energy $\left(\mathrm{E}_{\mathrm{g}}\right)$ of 0.0617 $0.0456 \mathrm{eV}$ was calculated. When compared to values observed by Mardare et al. 0.13-0.3 eV it becomes evident that that the observed band gap energy is unfortunately orders of magnitude lower. These values show that further investigation of the relation of $\mathrm{TiO}_{2}$ as an intrinsic semiconductive material is needed to consider temperature effects.
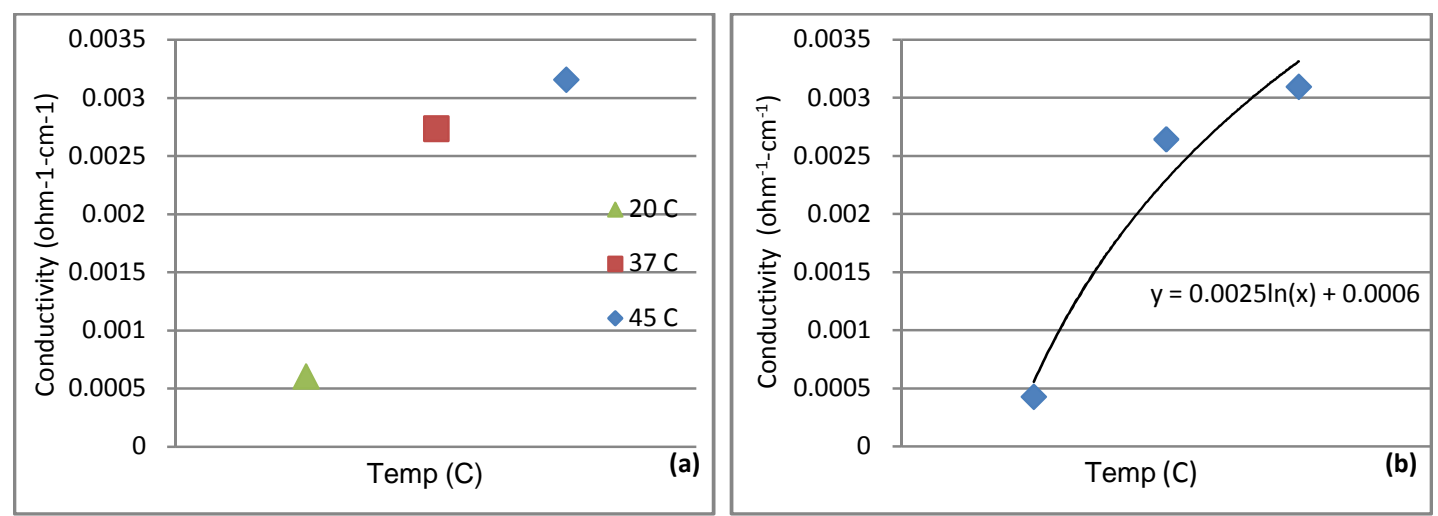

Figure 4. 4 (a)Conductivity measurements at 20, 37, and 45 C showing as temperature increase so does conductivity and (b) shows the best function of the plotted data used to calculate the $E_{g}$ values.

\subsection{C-AFM Experiments Compared to Oxide Film Growth Model}

As mentioned earlier, repassivation has been studied in detail on the macro and microscales. Such studies have developed oxide film growth models that have been theorized to model the 
kinetics of repassivation. Models such as the Ambrose model states that; as freshly exposed metal surfaces begin to grow an oxide film, the area fraction covered by the oxide, $\Theta$ increases with time (Ambrose, 1983). A similar model that can be used to describe this chemical reaction of oxide regeneration is the Langmuir adsorption model. In this model Langmuir assumed that on a given surface there are a certain number of binding sites per unit area and of these sites a certain number of adsorbate particles that can occupy these sites (Butt, Graf, \& Kappl, 2006). Therefore, in the case of repassivation or oxide regeneration, as the freshly exposed metal surface as in the performed experiments in this paper a created amount of binding sites becomes available to the available adsorbate in the system. However, the Langmuir model is based on ideal gasses and is primarily used with gases, but its principles of adsorption can be adopted in the case of repassivation reactions. 


\section{CONCLUSION}

It was the great Roman philosopher Pliney the Elder who first wrote about and studied the corrosion phenomena taking place at the surfaces of metals. Pliney first discovered and discussed that surfaces act as bodies that interact with each other and external agents (Roberge, 2008). Today much scientific advancement has been made toward the understanding of corrosion since Pliney's first discoveries and in this research paper, the stepping-stones for novel innovative studies of nanoscale repassivation was presented. This thesis incorporates sample preparation, nano-scratch test methods, and the characterization and analysis of repassivation of Ti-6Al-4V with the use of AFM techniques. As a result of this research, promising development of the use of AFM to study in-vitro repassivation has been gained. Although, the overall goal of studying repassivation on Ti-6Al-4V in-vitro with the characterization of conductance measurements was not fully accomplished, the results do provide proof of concept for further studies.

It was concluded that with the development of the created nano-scratch test methods in the IVAFM experiments, the AFM was capable of creating in-vitro scratches on the anodized oxide layers. Unfortunately, with the use of the z-sensor data, the AFM was not capable of observing repassivation after creating scratches, as the AFM scanner was determined to be too slow.

In the in-vitro C-AFM experiments, the AFM did not capture evidence of repassivation with the AFM conductive module after creating scratches in HBSS, but rather was affected by the excess of ions in the solutions creating amplified current measurements that the conductance module was unable to resolve. The C-AFM experiments performed in air however were capable of resolving current measurements post nano-scratch methods. Unfortunately, repassivation was unable to be observed with the performed techniques. The collected data rather indicates the observation of oxidation growth as the methods employed with the AFM were too slow to capture the instantaneous reactions. However, during these experiments observations of thermal effects with the current measurements were observed with each testing temperature. With the observed 
current measurement, the data was then able to observe temperature effects on conductivity and electron mobility during the performed testing conditions indicating that what was observed was representative of semiconductive properties. Incidentally, the collected data was also capable of measuring electron mobility and conductivity that was similar to other studied performed on $\mathrm{TiO}_{2}$ and shows evidence that conductive AFM is adequate to further explore the semi-conductive properties of $\mathrm{TiO}_{2}$.

Regarding the in-vitro repassivation experiments, the results of this research require further refinement to determine how to combine the ability of the AFM to create nano-scratches, as well as perform conductance measurements in-vitro. By combining these two abilities more accurate assessments of in-vitro repassivation kinetics can be achieved on the nanoscale to better understand repassivation and biological implications of repassivation corrosion for the prevention of late on-set infections.

Upon concluding this research one can say that repassivation of an implants protective layer is a great thing as it is the electrochemical process in which the protective layer is restored.

However, if a condition occurs where cyclic damage occurs to an implants protective oxide layer, a piece-wise condition of repassivation occurs. In this case, the piece-wise or cyclical condition of repassivation is better known as fretting corrosion or tribocorrosion. In this later state of tribocorrosion, biological implications can be detrimental. As cyclic electrochemical reactions occur over time, they can have the same unfavorable outcome as continuous corrosion. Resulting most often in, implant removal or unwanted implant failure. In general as described at the nano and macroscale, repassivation is a chemical reaction that occurs during the dissolution of a metal in a corrosive environment (e.g. human internal environment). These chemical reactions result in by-products such as the release of ions, the transfer of electrons, and eventually implant debris that can interact with the surrounding environment (host) and result in prolonged foreign body responses. These FBR's are the human body's natural response to foreign objects (e.g. ions, implant debris, \& implants) and the body will continue to try and remove these foreign bodies 
with immunological responses, macrophages, and protein encapsulation until the implant is removed. During the release of these immunological responses, infection can occur and with cyclic conditions of repassivation resulting in the accumulation of puss, infectious tissue, and implant debris. In the case of the sinus infection observed locally around loose screws in spinal fixation devices (Soultanis, Mantelos, Pagiatakis, \& Soucacos, 2003), this is most likely the cause of these sinus tracts, as a screw implant interface is an ideal location for fretting or tribocorrosion to occur. With cyclical compression cycles associated with normal walking biomechanics, spinal fixation devices can be subjected to stresses that can cause loosening of implant screws. Overtime as these cyclic stresses continue implant screws can become looser, affecting the tribological interaction between the two surfaces. As the screw continues to loosen the tribological effects progressively worsens from micro motion to macro-motion and particulate generation. At this state of particulate generation and ion dissolution infections can develop. Therefore, these sinus tract infections cannot be said to be directly caused by repassivation, but rather caused by a cascade of events that were initiated from repassivation and corrosion. Therefore, a better understanding of repassivation and corrosion at the nanoscale is needed to better understand the originating mechanisms of late on-set infections in patients with orthopaedic implants. 


\section{RECOMMENDATIONS FOR FUTURE WORK}

The work performed in this thesis was intended to develop AFM techniques for creating and characterizing nano-repassivation of Ti-6Al-4V in-vitro and in air. The following areas presented are intended to present some of the issues that came about during this study and which can be used to build upon for future research.

\subsection{Sample Fabrication}

\subsubsection{Polishing}

The use of metallography polishing techniques to prepare smooth flat samples in this study proved to have excessive variability in surface characteristics. The use of metallography polishing techniques did not provide samples that were consistently flat and polished to the same extent. When using AFM techniques, it becomes imperative that samples be as flat and smooth as possible as even the slightest of uneven samples can affect the AFM during use.

Identically prepared smooth samples with the use of micro fabrication techniques should be considered to control flatness, substrate morphology, oxide thicknesses, and allow for oxide measurability. With the use of microfabrication techniques, samples can all be prepared on a single silicon wafer with the ability to fabricate integrated circuits within in the samples that could also be used with conductive AFM experiments.

\subsubsection{Anodization}

With the use of electrochemical anodization methods, oxides were grown to a thickness to $\sim 15$ $20 \mathrm{~nm}$. As mentioned previously the prepared samples proved to be difficult to accurately measure oxide thicknesses prior to testing. The lack of microscopy techniques to measure such thin oxides in-house as well as accurate sources elsewhere resulted in undetermined oxide thicknesses prior to testing. The estimated oxide thicknesses were determined with literature 
equations and correlated growth methods (Sul, Johansson, Jeong, \& Albrektsson, 2001).

An accurate method of determining oxide thicknesses would have provided accurate starting oxide measurements prior to testing, allowing for verification that the scratch depths created with the AFM were indeed penetrating the entire oxide layer. The use of X-ray photo spectroscopy (XPS) could be used to accurately measure oxides before performing experiments. The use of XPS has been proven to be successful in other studies and should be considered for future experiments (Milosev, Hukovic, \& Strehblow, 2000).

\subsubsection{In-House Fabrication}

In an effort to minimize research costs, in-house fabrication of silicon cantilevers can be attempted. Using microfabrication techniques to pattern and etch silicon, cantilevers can be fabricated by the hundreds with each wafer. Identically prepared smooth samples with the use of micro fabrication techniques should be considered to control flatness, substrate morphology, oxide thicknesses, and allow for oxide measurability.

With the use of microfabrication techniques, samples can all be prepared on a single silicon wafer with the ability to fabricate integrated circuits within in the samples that could also be used with conductive AFM experiments. Utilizing the Rohwedder in the clean room, titanium can be sputtered on to a silicon wafer where the thickness can be controlled. After sputtering a uniform layer of titanium, controlled oxide growth methods can be performed to grow an oxide. Thermal oxidation can be attempted with the use of the tube furnaces as well as electrochemical methods in the wet bench. Using known oxide growth methods simple microfabrication techniques to measure oxide thickness can be utilized such as ellipsometry. This technique was originally attempted, but the non uniform oxide sample surfaces being too rough too much light was scattered affecting the measurements of refractive index that is used to determine the oxide thickness.

As the subject of investigation was repassivation at the nanoscale, it only makes sense to 
embrace the micro/nanofabrication abilities of samples to help better understand and emulate what actually occurs at the nanoscale.

\subsection{AFM Techniques}

Initially the goal of this research was to develop and perform in-vitro characterization methods for exploring repassivation surface effects from AFM scratch methods. Unfortunately, it was discovered that conductive AFM is extremely difficult to perform in a conductive fluid. Therefore the initial experiments were altered to allow the use of conductive AFM in air. Therefore, further research on developing a test method for performing conductive AFM scratch tests in HBSS is needed to decipher how to perform repassivation conductance measurements in a conductive media.

However, overall there were several advancements to this study: analysis of the ability to create in-vitro nano-scratch test methods for the characterization of repassivation with the use of AFM; and the ability to characterize repassivation with conductive AFM techniques in air. To improve the methods of measuring repassivation kinetics, some key additions can help to improve the kinetic observations. Coupling the existing AFM Bioheater Petridish accessory with a potentiostat to be able create and measure OCP's while performing nano-scratches with the AFM tip. Another method to improve the C-AFM experiments, would be to use a lock-in amplifier. The lock-in amplifier allows for accurate measurements to be taken when the original signal that is trying to be measured is obscured by other noise sources such as; frequency signals from electron and ionic transport as well as other outside signal sources. This will help with trying to resolve the nano-femto amps of current.

In this study, the use of the AFM allowed for the characterization of repassivation in new unique ways. To improve upon these methods, the use of cantilever tips with greater mechanical strength and durability would prove to be beneficial for manipulating the oxide surfaces. With the limited funding for this research cantilever tips were at a premium and often reused to extend the 
use of the cantilever. By having an abundant source of cantilevers, refined characterization can be achieved. The use of a nanoindentor to replicate the experiments performed in this paper may prove to be beneficial as the nanoindentor is a much more mechanically sturdy design for manipulating substrates on the nanoscale.

Additional AFM studies may further analyze in-vitro nano-repassivation behavior of Ti-6Al-4V oxide growth rates, behavior in the conductance of oxide regeneration in-vitro, and effects of electron mobility in oxides and how it affects repassivation in-vitro. With the use of AFM, more information about nano repassivation can be explored with refined techniques and should be combined with atomistic simulation. Through combining atomistic simulation with actual AFM data, virtual nano scratches can be created on substrates and then compared to AFM techniques. The combination of the two would allow for further development of nano manipulation for biomedical and biological research. 


\section{REFERENCES}

Abraham, F. (1974). Homogenous Nucleation. New York: Academic Press.

Aduda, B., Ravirajan, P., Choy, K., \& Nelson, J. (2004). Effect of morphology on electron dirft mobility in porous Tio2. International Journal of photoenergy, 141-147.

Akazawa, T., Minami, S., Takahashi, K., Kotani, T., Hanawa, T., \& Moriya, H. (2005). Corrosion of Spinal Implants Retrieved From Patients With Scoliosis. Orthopaedic Science, 200-205.

Ambrose, J. R. (1983). Repassivation Kinetics. In J. Scully, Treatise on materials science and technology, corrosion: Aqueous processes and passive films (pp. 175-204). NY: Academic Press.

Anderson, J. M., Rodriguez, A., \& Chang, D. T. (2008). FOREIGN BODY REACTION TO BIOMATERIALS. Semin Immunol , 20 (2), 86-100.

Antti-Poika, I., Jofefsson, G., Konttinen, Y., Lidgren, L., \& et.al. (1990). Hip arthroplasty infection. Current concepts. Acta Orthop Scand , 61, 163-169.

Ask, M., Lausmaa, \& Kasemo, B. (1989). Preparation and surface spectroscopic characterization of oxide films on Ti-6Al-4V. Applied Surface Science (35), 283-301.

(2006). Semiconductors. In D. Askeland, \& P. Phule, The Science and Engineering of Materials (pp. 697-700). Toronto: Thomson.

Asylum Reseach. (2008, October 15). Petri Dish Holder and Heater. Retrieved July 17, 2009, from Asylum Research The Technology Leader in Scanning Probe and Atomic Force Microscopy: http://asylumresearch.com/Products/PetriDishHolderHeater/PetriDishHolderHeater.shtml

Asylum Research. (2008). MicroAngelo. Retrieved June 10, 2009, from Asylum ResearchAtomic Force Microscopes:

http://www.asylumresearch.com/Applications/MicroAngelo/MicroAngeloPFMDSHR.pdf

Asylum Research. (2009). The technology leader in scanning probe and atomic force microscopy. Retrieved 2009, from Asylum Research ORCA:

http://asylumresearch.com/Applications/Orca/Orca.shtml

Brechignac, C., Houdy, P., \& Lahmani, M. (2006). Nanomaterials and Nanochemistry. Belin, France: Springer.

Butt, H., Graf, K., \& Kappl, M. (2006). Physics and Chemistry of Interfaces. Weinheim: Wiley$\mathrm{VCH}$.

Callister, W. D. (2003). Electron Mobility. In W. D. Callister, Materials Science and Engineering an Introduction (pp. 619-620). Hoboken, NJ: John Wiley \& Sons, Inc. 
Cambel, V., \& Soltys, J. (2007). The influence of sample conductivity on local anodic oxidation by the tip of atomic force microscope. JOURNAL OF APPLIED PHYSICS , 102, 1-7.

Contu, F., Elsener, B., \& Bohni, H. (2004). A study of potenials achieved during mechanical abrasion and the repassivation rate of titanium and Ti-6Al-4V in organic buffer sloutions and bovine serum. Electrochemica Atca , 50, 33-41.

Costerton, J. W., Cheng, K. J., Greesey, G. G., Ladd, T. I., Nickel, J. C., Dasgupta, M. M., et al. (1987). Bacterial biofilms in nature. Annual Review Microbiology , 41, 435-464.

Crozier, K. B., Yaralioglu, G. G., Degertekin, F. L., Adams, J. D., Minne, S. C., \& Quate, C. F. (2000). Thin film characterization by atomic force microscopy at ultrasonic frequencies. Applied physics letters, 1950-1952.

Eigler, D. M., \& Schweizer, E. K. (1990). Positioning single atoms with a scanning tunnelling microscope. Nature, 334, 524-526.

Fotiadis, D., Scheuring, S., Muller, S., Engel, A., \& Muller, D. (2002). Imaging and manipulation of biological structures with the AFM. Micron, 33, 385-397.

Gaine, W. J., Andrew, S. M., Chadwick, P., Cooke, E., \& Williamson, J. B. (2001). Late Operative Site Pain With Isola Posterior Instrumentation Requiring Implant Removal. Spine , 26, 583-587.

Geetha, M., Singh, A. K., Asokamani, R., \& Gogia, A. K. (2009). Ti Based Bionaterials, the Ultimate Choice for Orthopaedic Implants- A Review. Progress In Material Science , 54, 397 425.

Gilbert, J. L., Buckely, C. A., \& Lautenschlager, E. P. (1996). Titanium oxide film fracture and repassivation: The effects of potential, $\mathrm{pH}$ and aeration. In S. Brown, J. Lemons, S. Brown, \& J. Lemons (Eds.), Medical Applications of Titanium and Its Alloys: The Material and Biological Issues, ASTM STP 1272 (pp. 199-215). Ann Arbor, MI: ASTM STP 1272.

Goldberg, J. R., \& Gilbert, J. L. (1998). Electrochemical response of CoCrMo to high-speed fracture of its metal oxide using electrochemical scratch test methods. Journal of Biomedical Materials Research Part A , 37 (3), 421-431.

Gristina, A. G., \& Costerton, J. W. (1985). Bacterial adherence to biomaterials and tissue. The significance of its role in clinical sepsis. Journal of Bone and Joint Surgery , 67, 264-273.

Hallab, N. J., Jacobs, J. J., \& Katz, J. L. (2004). 7.7 Orthopedic Applications. In B. D. Ratner, A. S. Hoffman, F. J. Schoen, \& J. E. Lemons, Biomaterials Science An Introduction to Materials in Medicine (2nd ed., pp. 526-555). San Diego, CA, USA: Elsevier Academic Press.

Hallab, N., Merritt, K., \& Jacobs, J. J. (2001). Metal Sensitivity in Patients with Orthopaedic Implants. The Journal of Bone \& Joint Surgery, 80, 428-435.

Jacobs, J. J., Gilbert, J. L., \& Urban, R. M. (1998). Current Concepts Review - Corrosion of Metal Orthopaedic Implants. The Journal of Bone \& Joint Surgery , 80, 268-282. 
Jones, D. A. (1996). The Technology and Evaluation of Corrosion. In D. A. Jones, Principles and Prevention of Corrosion (Second ed., pp. 1-5). Upper Saddle River, NJ, USA: Prentice Hall.

Khan, M. A., Williams, R. L., \& Williams, D. F. (1999). Corrosion Behaviour of Ti-6Al-4V, Ti6Al-7Nb, and Ti-13Nb-13Zr in Protiens Solutions. Biomaterials , 631-637.

Khan, M. S., Rehman, S. u., Ali, M. A., Sultan, B., \& Sultan, S. (2008). Infection in Orthopedic Implant Surgery, Its Risk Factors and Outcome. Ayub Meidacl College Abbottabad , 20 (1), 2325 .

Khurshudov, A. G., Kato, K., \& Koide, H. (1996). Nano-wear of the diamond AFM probing tip under scratching of silicon, studied by AFM. Tribology Letter, 345-354.

Lazzarino, M., Mori, G., Sorba, L., Ercolani, D., Biasiol, G., \& al., e. (2006). Chemistry and formation process of $\mathrm{Ga}(\mathrm{Al}) \mathrm{As}$ oxide during local anodic oxidation nanolithography. Surface Science, 600, 3739-3743.

Lecuire, F., Gontier, D., Carrere, J., \& al., e. (2003). Ten-year surveillance of nosocomial surgical site infections in an orthopaedic surgery department. Rev Chir Orthop Repara-trice Appar Mot, $89,479-487$.

Long, P. H. (2008). Medical Devices in Orthopedic Applications. Toxicologic Pathology, 36 (1), 85-91.

Mardare, D., Baban, C., Gavrila, R., Modreanu, M., \& Rusu, G. (2002). On the structure, morphology and electrical conductivities of titanium oxide thin films. Surface Science , 468-472.

Mathew, M., Pai, P., Pourzal, R., Fischer, A., \& Wimmer, M. (2009). Significance of

Tribocorrosion in Biomedical Applications (Review Article). Advances in Tribology, 1-12.

Maurice, V., \& Marcus, P. (2009). Corrosion at the Nanoscale. In P. Schmuki, \& S. Virtanen, Electrochemistry at the Nanoscale (pp. 377-405). New York: Springer.

Maurice, V., Talah, H., \& Marcus, P. (1994). Surface Science .

Milosev, I., Hukovic, M. M., \& Strehblow, H. H. (2000). Passive Film on Orthopaedic TiAlV alloy Formed in Physiological Solution Investigated by XPS. Biomaterials , 2103-2113.

Mokross, B. (2003). Nucleation Theory and Small Systems Thermodynamics.

Material.Phys.Mech, 13-20.

Mu, Y., Kobayashi, T., Sumita, M., Yamamoto, A., \& Hanawa, T. (2000). Metal Ion Release From Titanium with Active Oxygen Species Gereated by Rat Macrophages in-vitro. Biomedical Materials Research, 238-243.

Ponseti, I. V. (1991). History of Orthopaedic Surgery. Iowa Orhtopedic Journel , 59-64.

Richards. (1995). Delayed infectionsfollowing posterior spinal instrumentation for the treatment of idiopathic scoliosis. Journal of Bone and Joint Surgery , 77A, 524-529. 
Roberge, P. R. (2008). Corrosion Engineering Principles and Practice. New York: Mc Graw Hill.

Rugar, D., Mamin, H. J., Guethner, P., Lambert, S. E., Stern, J. E., \& et.al. (1990). Magnetic force microscopy: general principles and application to longitudinal recording media. J. Appl. Phys , 68 (3), 1169-1183.

Santinacci, L., Zhang, Y., \& Schmuki, P. (2005). AFM scratching and metal deposition through insulating layers on silicon. Surface Science, 597, 11-19.

Schultz, J. W., \& Hassel, A. W. (2003). Passivity of Metals, Alloys, and Semiconductors. In M. Stratmann, \& G. S. Frakel, Encyclopedia of Electrochemistry Corrosion and Oxide Films (pp. 216-267). KGaA Weinham: Wiley-VCH.

Sculo, T. P. (1993). The economic impact of infected total joint arthoplasty. AAOS , 42, 349-351.

Soultanis, K., Mantelos, G., Pagiatakis, A., \& Soucacos, P. (2003). Late Infection in Patients With Scoliosis Treated With Spinal Instrumentation. Clinical Orthopaedics and Related Research, 411, 116-123.

Spiegelman, F. (2006). Electronic Structure in Cluster and Nanoparticles. In C. Brechignac, P. Houdy, \& M. Lahmani, Nanomaterials and Nanochemistry (pp. 135-195). Belin: Springer.

Stinchfield, F. E., Bigliani, L. U., Neu, H. C., Goss, T. P., \& et.al. (1980). Late hematogenous infection of total joint replacement. Journal of bone and joint surgery, 62, 1345-1350.

Sul, Y.-T., Johansson, C. B., Jeong, Y., \& Albrektsson, T. (2001). The Electrochemical Oxide Growth Behaviour on Titanium in Acid and Alkaline electrolytes. Medical Engineering and Physics , 23, 329-346.

Takao, H. (2002). Evaluation techniques of metallic biomaterials in vitro. Science and Technology of Advanced Materials , 3, 289-295.

Temenoff, J. S., \& Mikos, A. G. (2008). Biomaterial Degradation. In J. S. Temenoff, \& A. G. Mikos, Biomaterials The intersection of Biology and Materials Science (pp. 177-190). Upper Saddle River, NJ, USA: Pearson Prentice Hall.

Tezer, M., Kuzgun, U., Hamzaoglu, A., Ozturk, C., Kabukcuoglu, F., \& Sirvanci, M. (2005). Intraspinal Metalloma Resulting in Late Paraparesis. Archives of Orthopedic Trauma Surgery, 417-421.

Trampuz, A., \& Zimmerli, W. (2006). Diagnosis and Treament of Infections Associated with fracture Fixation Devices. Injury, 59-66.

Van de Belt, H., Nuet, D., Schenk, W., Van Horn, J. R., Van der Mei, H. C., \& Busscher, H. J. (2001). Infection of Orthopedic Implants and The Use of Antibiotic-loaded Bone Cements. Acta Orthopaedica, 557-571.

Wang, S., Currier, B. L., \& Yaszemski, M. J. (2006). Orthopedic Prostheses and Joint Implants. In S. A. Guelcher, \& J. O. Hollinger, An Introduction to Biomaterials (pp. 369-375). Boca Raton, FL, USA: Taylor \& Francis Group. 
Weisenhorn, A. L., Hansma, P. K., Albrecht, T. R., \& Quate, C. F. (1989). Forces in atomic force microscopy in air and water. Applied Physics , 54, 2651-2653.

Yasushi, T., Yasuyuki, S., Genta, W., Tsutomu, Y., \& Jun-ichi, S. (2007). Measurement of faradaic current during AFM local oxidation. Journal of Physics, 1147-1151.

Zorba, S., Watkins, N. J., Yan, L., \& Gao, Y. (2001). Current-Voltage Measurements as a Function of Applied Tip Force on Pentacene by Conducting Probe Microscopy. Mat. Res. Soc. Symp. Proc, 660. 